\title{
Experimental Investigation of Megavoltage Electron Beam Scattering
}

by

\section{Andrew F. McDonald}

\author{
A thesis submitted to the \\ Faculty of Graduate Studies and Research \\ in partial fulfillment of the requirements \\ for the degree of \\ Master of Science \\ Department of Physics \\ Carleton University \\ Ottawa-Carleton Institute of Physics \\ Ottawa, Canada
}

May 15, 2008

Copyright (C) 2008 Andrew F. McDonald 


$\begin{array}{ll}\begin{array}{l}\text { Library and } \\ \text { Archives Canada }\end{array} & \begin{array}{l}\text { Bibliothèque et } \\ \text { Archives Canada }\end{array} \\ \begin{array}{l}\text { Published Heritage } \\ \text { Branch }\end{array} & \begin{array}{l}\text { Direction du } \\ \text { Patrimoine de l'édition }\end{array} \\ \begin{array}{l}\text { 395 Wellington Street } \\ \text { Ottawa ON K1A 0N4 } \\ \text { Canada }\end{array} & \begin{array}{l}\text { 395, rue Wellington } \\ \text { Ottawa ON K1A 0N4 } \\ \text { Canada }\end{array}\end{array}$

Your file Votre référence ISBN: 978-0-494-40660-1

Ourfile Notre référence

ISBN: 978-0-494-40660-1

NOTICE:

The author has granted a nonexclusive license allowing Library and Archives Canada to reproduce, publish, archive, preserve, conserve, communicate to the public by telecommunication or on the Internet, loan, distribute and sell theses worldwide, for commercial or noncommercial purposes, in microform, paper, electronic and/or any other formats.

The author retains copyright ownership and moral rights in this thesis. Neither the thesis nor substantial extracts from it may be printed or otherwise reproduced without the author's permission.
AVIS:

L'auteur a accordé une licence non exclusive permettant à la Bibliothèque et Archives Canada de reproduire, publier, archiver, sauvegarder, conserver, transmettre au public par télécommunication ou par l'Internet, prêter, distribuer et vendre des thèses partout dans le monde, à des fins commerciales ou autres, sur support microforme, papier, électronique et/ou autres formats.

L'auteur conserve la propriété du droit d'auteur et des droits moraux qui protège cette thèse. $\mathrm{Ni}$ la thèse ni des extraits substantiels de celle-ci ne doivent être imprimés ou autrement reproduits sans son autorisation.
In compliance with the Canadian Privacy Act some supporting forms may have been removed from this thesis.

While these forms may be included in the document page count, their removal does not represent any loss of content from the thesis.
Conformément à la loi canadienne sur la protection de la vie privée, quelques formulaires secondaires ont été enlevés de cette thèse.

Bien que ces formulaires aient inclus dans la pagination, il n'y aura aucun contenu manquant.

\section{Canada}




\begin{abstract}
This work set out to measure high accuracy electron scattering profiles for use in comparisons to Monte Carlo simulations. Electron scattering profiles for various elemental scattering foils at different mass thicknesses ranging from 20 to $220 \mathrm{mg} / \mathrm{cm}^{2}$ and incident beam energies of 13 and $20 \mathrm{MeV}$ were measured. The experimental profiles were characterized using the characteristic angle $\left(\theta_{c}\right)$, the angle at which the intensity is $1 / e$ the peak intensity. The characteristic angle ranged from $1.57^{\circ}$ for the thinnest titanium alloy to $8.55^{\circ}$ for the thickest gold foil. Investigations of various initial beam parameters, set-up geometry and detectors were also performed.

Monte Carlo simulations of the experimental measurements were performed. Differences were found between the measured and calculated characteristic angles ranging from $-0.5 \%$ for the thickest titanium alloy to $-9.0 \%$ for the thinnest titanium alloy. While there are these differences between the measured and calculated profiles, the measured electron scattering profiles, with uncertainties under $1 \%$, are excellent for further use in Monte Carlo validation.
\end{abstract}




\section{ACKNOWLEDGEMENTS}

Firstly, I would like to thank my co-supervisors Dr. Carl Ross and Dr. Malcolm McEwen for their guidance, support and especially patience during this work.

Also, the rest of the Ionizing Radiation Standards group for all their help, both academically and otherwise. David Marchington and Matt Kosaki for aid in the design and operation of the experimental measurements. To Ernesto Mainegra-Hing, Iwan Kawrakow, and Blake Walters for help with the Monte Carlo simulations. To all the rest in the group who made my time at National Research Council an enjoyable one too, thank you.

I would like to acknowledge my grandfather, who had a constant bright outlook on life and taught me to enjoy the finer things in life. I would like to thank my family, Mom, Dad and Chris for your encouragement. Now I'm one of you! To my friends, for helping me to keep my sanity. And Jaelle, you have always been there for me on this journey. 


\section{TABLE OF CONTENTS}

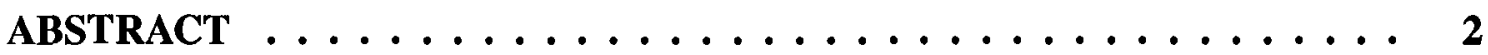

LIST OF TABLES .......................... 6

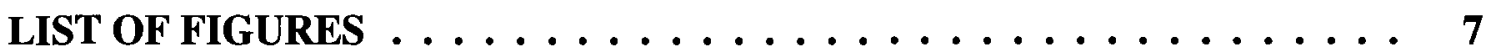

CHAPTER 1 Introduction $\ldots \ldots \ldots \ldots \ldots$

CHAPTER 2 Theoretical Background and Literature Review . . . . . . . . 4

2.1 Theoretical Background . . . . . . . . . . . . . . . 4

2.2 The Monte Carlo Technique . . . . . . . . . . . . . . 7

2.3 Literature Review and Experimental Design . . . . . . . . . . . 9 9

2.4 Linear Accelerators . . . . . . . . . . . . . . . . . . 15

2.4.1 Definitions of beam characteristics . . . . . . . . . 15

2.4.2 Rationale for use of the Vickers linear accelerator . . . . . . . . 15

2.5 Influence Quantities . . . . . . . . . . . . . . . . . 18

CHAPTER 3 Experimental Set-up and Measurements . . . . . . . . . . 22

3.1 Materials and Methods . . . . . . . . . . . . . . 22

3.2 Initial Measurements . . . . . . . . . . . . . . . 30

3.3 Scattering Foils . . . . . . . . . . . . . . . . 30

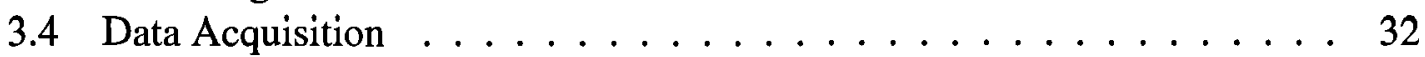

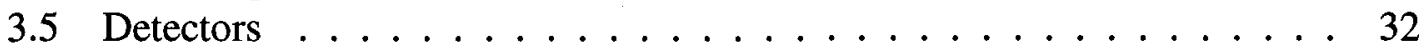

CHAPTER 4 Monte Carlo Simulations $\ldots \ldots \ldots \ldots \ldots$

4.1 Monte Carlo Codes . . . . . . . . . . . . . . . . . 36

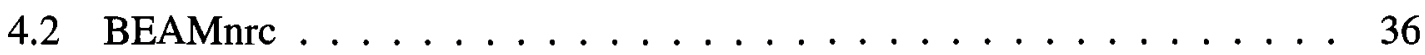

4.3 Other EGSnrc Tools . . . . . . . . . . . . . . . . . . . 40

CHAPTER 5 Results and Discussion . . . . . . . . . . . . . . 42

5.1 Analysis of the Data . . . . . . . . . . . . . . . 42

5.2 How the Characteristic Angle was Extracted . . . . . . . . . . . . 44

5.2 .1 Peak of the distribution . . . . . . . . . . . . . 45

5.2.2 Sensitivity of characteristic angle over data range . . . . . . . 47

5.3 Beam Characteristics ... . . . . . . . . . . . . . 48

5.4 Spatial Resolution . . . . . . . . . . . . . . . 53 
5.5 Corrections for Influence Quantities $\ldots \ldots \ldots \ldots \ldots \ldots$

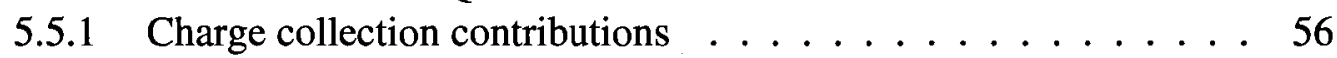

5.5 .2 Secondary radiation $\ldots \ldots \ldots \ldots \ldots$

5.6 Uncertainties . . . . . . . . . . . . . . . . 62

5.7 Results . . . . . . . . . . . . . . . . . 66

5.8 Discussion . . . . . . . . . . . . . . . . . . . . 69

CHAPTER 6 Conclusions $\ldots \ldots \ldots \ldots \ldots \ldots \ldots \ldots \ldots$

References . . . . . . . . . . . . . . . . . 76 


\section{LIST OF TABLES}

2.1 Summary of four commonly used dosimetric systems . . . . . . . . . . 14

3.1 Mass thickness of the scattering foils . . . . . . . . . . . 31

3.2 Detectors investigated . . . . . . . . . . . . . 35

4.1 Input parameters used in BEAMnrc calculations . . . . . . . . . . . . 39

4.2 Input parameters used in DOSRZnrc and FLURZnrc calculations . . . . . 41

5.1 Monte Carlo investigation of beam diameter . . . . . . . . . . . 51

5.2 Uncertainties of characteristic angle .............. 66

5.3 Measured characteristic angle at $13.0 \mathrm{MeV} \ldots \ldots$. . . . . . . . 67

5.4 Measured characteristic angle at $20.0 \mathrm{MeV} \ldots \ldots$. . . . . . . . . 68

5.5 Additional angle required to reduce difference between Monte Carlo calculations and experimental measurements . . . . . . . . . . . 70 


\section{LIST OF FIGURES}

2.1 Mass scattering powers for elements of interest $\ldots \ldots \ldots 17$

3.1 Experimental hall of the Vickers linear accelerator . . . . . . . . . . . 23

3.2 Schematic of the Experimental set-up . . . . . . . . . . . . . 24

3.3 Concentration of oxygen in aluminized Mylar bag during flushing . . . . 27

3.4 Photo of experimental set-up on axis . . . . . . . . . . . . 28

3.5 Photo of experimental set-up sideview . . . . . . . . . . . . . . 29

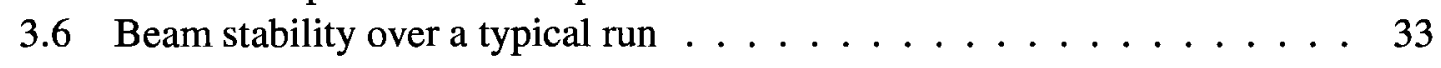

4.1 BEAMnrc input source routine .................... 38

5.1 Typical experimental measurement . . . . . . . . . . . . . 43

5.2 Plot for extraction of the characteristic angle . . . . . . . . . . 46

5.3 Experimental beam profile measurement . . . . . . . . . . . . . . . 49

5.4 Monte Carlo beam energy investigation . . . . . . . . . . . 51

5.5 Monte Carlo investigation mean angular spread . . . . . . . . . . 52

5.6 Investigation of spatial resolution . . . . . . . . . . . . 54

5.7 Polarity effect of the A16 ion chamber . . . . . . . . . . 58

5.8 Monte Carlo investigation of bremsstrahlung . . . . . . . . . . 60

5.9 Monte Carlo investigation of the energy fluence of both electrons and photons ............................ 61

5.10 Monte Carlo investigation of characteristic angle with increased foil

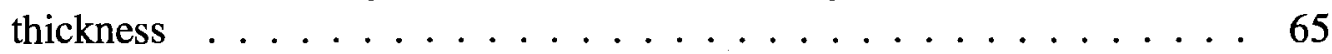

5.11 Scaled comparison of experiment measured to Monte Carlo calculations . $\quad 72$ 


\section{CHAPTER 1}

\section{Introduction}

Cancer is an ongoing concern for everyone, with approximately 1 in 4 Canadians dying from it (Canadian Cancer Society, 2007). Treatment of cancer is currently carried out using chemotherapy, radiation therapy and surgery and these three modalities are frequently used in tandem. Radiation therapy makes up $40 \%$ of the treatments, the majority by way of photon or electron radiation. A radiation treatment plan is designed to maximize the damage to cancer cells and minimize damage to healthy cells in the same region.

With different types of radiation and modes available to deliver the treatment, a treatment plan is an important step in the process. Some different types of radiation therapy are: external beam - use of a clinical linear accelerator (linac); brachytherapy - use of sealed implants or seeds within the body for treatment; and unsealed sources - a soluble radioactive substance used based on biological properties (for example, iodine is selectively taken up by the thyroid gland). Before treatment starts, a radiation therapy team determines the amount and type of radiation to be delivered. The radiation therapy team is comprised of a radiation oncologist, a doctor who specializes in radiation cancer treatment; a dosimetrist, who determines the proper radiation dose based on instructions by the radiation oncologist; a medical radiation physicist, who maintains the treatment machine and ensures the proper amount of radiation is delivered to correct areas of the body; and a radiation therapist, who delivers the radiation treatment.

For external beam radiation therapy, the treatment plan is set out by the radiation therapy team prior to treatment. The radiation therapy team will use a computed tomography (CT) scanner to take internal images of the patient which are used to plan the treatment. 
The radiation oncologist uses these CT images to contour (trace) the tumor and regions at risk and then apply a prescribed dose to the tumor. The contoured images are then used by a dosimetrist to plan the configuration of the treatment, i.e., number of beams and angles of incidence, energy, size of beams. A calculation of the treatment occurs. This calculation is based on pre-calculated beamlets (small, $1 \mathrm{~cm}$ by $1 \mathrm{~cm}$ beams) and approximations that are based on how particles will interact in the body regions and organs. The calculation usually takes 30 seconds for "simple" cases, and the dose distributions are verified by the radiation oncologist and radiation physicist. When the dose distribution is acceptable, then the radiation oncologist and radiation physicist approve the treatment plan. The patient returns to the centre and treatment occurs. The treatment takes place over the course of the 6-week fractionated treatment.

To overcome the approximations during the treatment plan, an exact simulation of the plan would be desirable. One option for this is state-of-the-art computer modeling technique known as Monte Carlo simulation.

Radiation transport using Monte Carlo simulations has been used for over 30 years in particle transport to model linear accelerators and also can be used to calculate dose to matter (Berger \& Seltzer, 1973). These Monte Carlo calculations have been compared to measured experimental results for benchmarking. Benchmarking of Monte Carlo simulations is required to ensure that the Monte Carlo calculations are able to model a true physical process. For benchmarking, current systems have been using experimental electron scattering distribution data from the 1950s (Hanson et al., 1951). While these measurements were carried out very carefully and accurately, having a comprehensive data set, for example, over a range of elements, thicknesses and energies, would be a more thorough approach for Monte Carlo verification.

This study is part of a larger collaborative project with Dr. Bruce Faddegon at the Uni- 
versity of California in San Francisco (UCSF). This National Institutes of Health (NIH) funded collaboration aims to create accurate and easy to commission radiotherapy beam models for use in Monte Carlo treatment planning systems. These beam models will provide the same level of precision and accuracy as the Monte Carlo systems but will be many times faster than the current commissioning routine. These beam models will be based on some key measurements from the clinical linac during the commissioning routine. The beam models will be developed for electron beams, based on experimental measurements from a research linac as well as instruments from three major manufacturers. A beam model in the context of Monte Carlo treatment planning is any algorithm that delivers the location, direction, and energy of particles to the patient dose-calculating algorithm (Chetty et al., 2007).

The goal of this study is to create a high precision experimental measurement of electron scattering distributions with uncertainty better than $1 \%$ level from thin foils in the clinical energy range. This dataset will include a range of elements from a titanium alloy to gold, of various mass thicknesses and two beam energies.

All of the work contained in this thesis has been performed by the author with exception of:

- measurement of the bremsstrahlung with the electromagnet,

- third person confirmation of the Monte Carlo simulations,

- day-to-day power up of the linear accelerator which was performed by trained personnel,

- investigation of the dose-rate dependence. 


\section{CHAPTER 2}

\section{Theoretical Background and Literature Review}

\subsection{Theoretical Background}

The knowledge of high-energy electron interactions is fundamental to the measurement of dose to a medium. Dose is the amount of energy deposited per unit mass by ionizing radiation. These high-energy electrons are known as ionizing radiation because, as the electrons pass through matter, they ionize atoms. There are three main interactions of importance in the energy range of hundreds of $\mathrm{keV}$ to $50 \mathrm{MeV}$. First, collisions with atomic electrons; second, bremsstrahlung also known as radiative losses; and, third, elastic scattering from the nuclei. These interactions are fundamental when discussing the characteristics of the electron beams and how electrons travel through matter.

Collisional interactions of incident electrons within a medium are the principal mechanism for energy loss. As the electron travels in a medium, a path of ionizations (and/or excitations) is created. The electron loses energy by way of Coulomb interactions with bound atomic electrons. The classical model of the interaction will be considered. As the electron passes close to an atom at a distance $b$, called the impact parameter, the electron interacts with the Coulomb field of the atom. These interactions will occur with a certain probability known as the cross section of interaction. These collisions are categorized into two types, soft and hard interactions. A soft collision occurs when the electron passes the atom at a relatively large distance. This interaction only causes a small effect on the Coulomb field of the atom and a small amount of energy is lost. For a hard collision, the electron is relatively close to the atom and interacts with a bound atomic electron. 
Occasionally, the electron causes this bound electron to be ejected from the atom.

Radiative losses, or bremsstrahlung, can also occur as electrons travel through matter. Bremsstrahlung is the process by which an electron accelerates past another charged particle such as another electron or an atomic nucleus, and gives off photons. The probability for electron-electron bremsstrahlung interaction is so small compared to the nuclear bremsstrahlung that it is not taken into account in most bremsstrahlung situations (Haug \& Nakel, 2003).

Energy loss by the electron beam is known as total mass stopping power and is defined by Equation 2.1. Total stopping power can be defined as the energy lost, $\mathrm{d} E$, per unit mass presented to unit area of the beam $(\rho \mathrm{d} l)$ :

$$
\frac{S}{\rho}=\frac{1}{\rho}\left(\frac{\mathrm{d} E}{\mathrm{~d} l}\right)
$$

where $\rho$ is the material density and $\mathrm{d} l$ is an infinitesimal thickness. Stopping power (S) and scattering power $(\mathrm{T})$ are material-dependent. To easily compare these properties over a wide range of materials the density is used for normalization. There are two components to stopping power: collision $\left((S / \rho)_{c o l}\right)$ and radiative $\left((S / \rho)_{\text {rad }}\right)$ events:

$$
\frac{1}{\rho}\left(\frac{\mathrm{d} E}{\mathrm{~d} l}\right)_{t o t a l}=(S / \rho)_{t o t}=(S / \rho)_{c o l}+(S / \rho)_{\text {rad }} .
$$

Conventional stopping power units are $\frac{\mathrm{MeVcm}^{2}}{g}$.

When an electron passes within the atomic radius, it interacts with the nucleus directly and will be scattered. This process is known as elastic nuclear scattering. The electron retains almost all of the initial energy and is only redirected, so the scattering is elastic. The mass scattering power $(T / \rho)$ is defined (ICRU report 35,1984$)$ as the mean square angle of scattering $\overline{\mathrm{d} \theta^{2}}$ per unit of mass thickness $\rho \mathrm{d} l$ : 


$$
\frac{T}{\rho}=\frac{1}{\rho} \frac{\overline{\mathrm{d} \theta^{2}}}{\mathrm{~d} l}
$$

Conventional mass scattering power units are $\frac{\text { radians }^{2} \mathrm{~cm}^{2}}{g}$.

This holds true for infinitesimal thickness slabs and is still used in practice for finite slab thicknesses. Rossi \& Greisen (1941) developed a different expression used for calculation of mass scattering powers within a medium, assuming small and independent deflections. This expression, Equation 2.4, is now used to calculate mass scattering powers as it has a more accurate formulation of the nuclear screening effect than the one previous defined in ICRU report 21, 1972.

$$
\frac{T}{\rho}=\pi\left(\frac{2 r_{e} Z}{(\tau+1) \beta^{2}}\right)^{2} \frac{N_{A}}{M_{A}}\left\{\ln \left[1+\left(\frac{\theta_{m}}{\theta_{\mu}}\right)^{2}\right]-1+\left[1+\left(\frac{\theta_{m}}{\theta_{\mu}}\right)^{2}\right]^{-1}\right\}
$$

where: $r_{e}$ - classical radius of an electron $\left(=1 / 4 \pi \epsilon_{0} \cdot e^{2} / m_{e} c^{2}\right), \epsilon_{0}$ - permittivity of free space, $m_{e} c^{2}$ - rest mass of an electron, $\mathrm{c}$ - speed of light in a vacuum, $\mathrm{Z}$ - atomic number, $\tau$ - ratio of kinetic energy to rest mass of the electron, $\beta$ - velocity of electron relative to velocity of light in a vacuum, $N_{A}$ - Avogadro constant, $M_{A}$ - molar mass of substance, A - mass number, $\theta_{m}$ - the cut-off angle due to the finite size of the nucleus, given in Equation 2.5 and $\theta_{\mu}$ - the screening angle, the screening of the nucleus due to the orbital electrons, given in Equation 2.6.

$$
\begin{gathered}
\theta_{m}=\frac{2 A^{-1 / 3}}{\alpha \beta(\tau+1)} \\
\theta_{\mu}=1.130 \frac{\alpha Z^{1 / 3}}{\beta(\tau+1)}
\end{gathered}
$$


where $\alpha$ is the fine structure constant $(\approx 1 / 137)$ and all other parameters have been defined previously.

There is a spectrum of scattering angles. However, the scattering can be categorized into two processes depending on the impact parameter from the nucleus: multiple smallangle scattering or single large-angle scattering. In both cases, many instances of scattering can occur. Multiple small-angle scattering is due to many small-angle scatterings which will result in the final deflection angle of the electron. While single large-angle scattering does not mean that the electron is scattered only once, but while the electron is traveling through the medium, a rare large-angle scattering event will occur and will result in the final large deflection angle away from the initial trajectory.

If the distance between the electron and the nucleus is relatively large then the electron interacts minimally with the nucleus and deflects at a small angle. With small-angle scattering, the interactions as the electron transverses the material tend to average out over the large number of scattering events and the final deflection of the many smallangle scatterings appears as a Gaussian distribution. Multiple small-angle scattering is highly analogous to diffusion, and the terms multiple scattering and diffusion are used interchangeably in many contexts. For example, optical elements designed to produce multiple scattering are also known as diffusers.

\subsection{The Monte Carlo Technique}

The Monte Carlo technique is used to model physical processes depending on the probabilities of occurrence. For example, a random walk would use a random number generator and from this number a movement would be performed in a certain direction depending on the probability of movement in that direction. In radiation transport, the Monte Carlo technique has been used in a similar way; however, in this case, the probability of the 
movement of the particles is contingent on the cross-section of interaction. In Monte Carlo simulations, there are four main components: i) the cross-section data for all the interactions, ii) the method of particle transport, iii) the physical geometry of the problem, and iv) the analysis of the result obtained from the simulation. This technique is very useful in simulating physical processes with a large number of events and can even show insight into some situations which cannot be studied experimentally such as interactions within a certain constituent of the simulation or tracking particles after a certain interaction (Rogers \& Bielajew, 1990).

Since the 1960s, the Monte Carlo technique has been used in radiation transport of particles for various applications and the technique has been ever increasing in terms of developments and uses (Rogers, 2006). Two of these applications are to model clinical linear accelerators in radiation treatment (Rogers et al., 1995) and to calculate dose to matter (Nelson et al., 1985). The use of Monte Carlo calculations in medical physics has developed to the point where Monte Carlo correction factors are used daily in radiation dosimetry. Using a Monte Carlo-based treatment planning system has been understood to be the most accurate dose calculation technique but in the past the time required for these simulations was longer than the conventional treatment plan system. Therefore, until recently, a Monte Carlo-based treatment planning system was not a viable option. However, the improvements in faster Monte Carlo codes, increase in CPU computing power and overall decrease in computer costs have led to the development of Monte Carlobased treatment planning systems.

There are many Monte Carlo codes for use in radiation transport, with a wide range of areas for use in research. These codes deal with the four main components (cross-section, transport, geometry and analysis) differently, but the outcome is still very similar. Some examples of these codes are: Electron Gamma Shower [the latest version is also known 
as EGSnrc (Kawrakow \& Rogers, 2000)], which focuses on the transport of electrons and photons; GEANT (Agostinelli et al., 2003), developed at CERN for use of simulating interactions from many types of particles; MCNPX 2002, used to simulate transport of many type of particles including neutrons; and PENELOPE (Salvat et al., 2003), used for electron-photon transport.

\subsection{Literature Review and Experimental Design}

The current experimental data for electron scattering distributions is somewhat limited.

Kulchitsky \& Latyshev (1942) performed electron scattering measurements over a range of materials using a $2.25 \mathrm{MeV}$ pencil beam of electrons. The materials ranged from aluminum to lead but for only one thickness of each. While this work had well defined experimental measurements, it was not in the typical clinical regime of $5-20 \mathrm{MeV}$. The electron beam had a diameter of $2.0 \mathrm{~mm}$ with a mean angular spread of about $0.5^{\circ}$. The target was set up in a very good vacuum drift region. The thickness of the targets was chosen to give a measured profile HWHM (half width at half the maximum intensity) of $\approx 10^{\circ}$. This set does not investigate the effect of the increase of foil thickness on the profile. The widths of the profiles are available in tabular format as the half-width where the intensity diminished by $1 / e$ with an experimental accuracy of 3-4 percent. Profiles are only presented as normalized relative values not absolute profiles. The dose per incident electron or fluence per incident electron (not the normalized values) are used in an absolute profile.

Ten years later, Hanson et al. (1951) performed electron scattering measurements for a $15.7 \mathrm{MeV}$ electron beam on both gold and beryllium thin foils. This was carried out in the clinical linacs' energy range but profiles for only two scattering foils of each element were measured. The electron beam had a beam diameter of $2.0 \mathrm{~mm}$ and a full angular 
width of $1^{\circ}$. Two gold foils $\left(18.66 \mathrm{mg} / \mathrm{cm}^{2}\right.$ and $\left.37.28 \mathrm{mg} / \mathrm{cm}^{2}\right)$ and two beryllium foils $\left(257.0 \mathrm{mg} / \mathrm{cm}^{2}\right.$ and $\left.491.4 \mathrm{mg} / \mathrm{cm}^{2}\right)$ were investigated using an ion chamber and magnetic analyzer set-up. The target was set up in a vacuum chamber. An adjustable aperture was located after a $10 \mathrm{~cm}$ drift region. This aperture was scanned across the width of the vacuum chamber as the detector for the profile. The electrons then passed through a $75^{\circ}$ magnetic analyzer. This reduced the scatter and bremsstrahlung and gave an energy resolution of $6 \%$ to the detected electrons. The widths of the profiles are available in tabular format as the angle at which the intensity is reduced to $1 / e$ the maximum intensity. The large-angle scattering region was also studied. The ratio of the two gold foils were used to investigate the boundary where small-angle and large-angle scattering occurs. This experimental electron scattering data has been used for benchmarking of Monte Carlo simulations (see discussion below).

Another electron scattering investigation was performed by Kovalev et al. (1972) for a range of energies and scattering foils. This experiment investigated tungsten, cadmium and copper scattering foils over a range of thicknesses at $12.8 \mathrm{MeV}, 18 \mathrm{MeV}$ and $25 \mathrm{MeV}$. The electron beam diameter at the scattering foil was $6 \mathrm{~mm}$ and the drift region was air. A thimble ion chamber detector was scanned in an arc at $13 \mathrm{~cm}$ from the scattering target. The ion chamber had a sensitive volume of $0.25 \mathrm{~cm}^{3}$ and angular dimension of $4.6^{\circ}$. During the measurements, the ion chamber recorded the current from the scattered electrons as well as the bremsstrahlung from the scattering foil. However the bremsstrahlung was subtracted to give only the scattered electrons, based on the measured bremsstrahlung current. Unfortunately, profile data could not be obtained from the paper as the resolution of the printed profiles was poor.

Blais \& Podgorsak (1992) used electron scattering profiles to determine the kinetic energy of clinical electron beams. Using a clinical accelerator with a few additions, Blais 
\& Podgorsak measured in-air electron scattering profiles for a range of set-ups. While Blais \& Podgorsak were measuring electron scattering profiles similar to the other studies, they did not look at various foil thicknesses or elements, only at determining the electron energy. A lead sheet with an aperture was mounted $65.1 \mathrm{~cm}$ from the linear accelerator scattering foil to provide a pencil-beam. To ensure the best set-up, the aperture and the thickness of the lead sheet were investigated. The aperture was varied from 1 to $3 \mathrm{~mm}$ in diameter and the lead sheet from 2 to $10 \mathrm{~mm}$ in thickness. The profiles were measured using radiographic films and the distance from the aperture to the film was also varied, from 30 to $60 \mathrm{~cm}$. The resolution of the film measurement was $0.8 \mathrm{~mm}$. Due to the use of a lead sheet in the set-up, transmitted electrons and bremsstrahlung photons from the lead sheet are also measured in the profile measurements. Therefore, this background measurement was subtracted from the overall measurements to give the profile. Blais $\&$ Podgorsak concluded that the variation in aperture diameter and lead thickness resulted in little to no change in the profile of the beam. As the distance from the source to measurement plane increases one is able to obtain the electron kinetic energy. This technique was compared and found to be consistent to other methods (the practical range and $R_{50}$, the point at which the depth-dose has reached $50 \%$ its maximum value) for electron beam energy determination.

No discussion of profile measurement uncertainties was provided for any of these studies. Therefore, no notion of the measured uncertainty on the profile or the characteristic angle is available for Monte Carlo comparison.

A number of studies have used these experimental data sets for comparison of electron scattering profiles to Monte Carlo calculations [Li \& Rogers (1995), Vilches et al. (2007)]. Li \& Rogers (1995) investigated mass scattering powers resulting from Monte Carlo calculations versus analytical calculations and also how these techniques compare 
to the experimental data set from Hanson et al. (1951). The Monte Carlo calculations agree with the experimental results whereas the ICRU report 35 (1984) Gaussian fit underestimates the mean square scattering angle.

Vilches et al. (2007) used the experimental data sets of Hanson et al. (1951) and Kulchitsky \& Latyshev (1942) to perform a study of some of the Monte Carlo radiation transport simulation packages. The packages used were PENELOPE [both v. 2003 (Salvat et al., 2003) and v. 2005 (Salvat et al., 2005)], GEANT3 (CERN Program Library Long Write-up, 1993), GEANT4 (Agostinelli et al., 2003), EGSnrc (Kawrakow \& Rogers, 2000) and MCNPX (RSICC Computer Code Collection, 2002). Vilches et al. reanalyzed the experimental data using the standard characteristic angle and also the intensity of the profile. Both were compared to Monte Carlo calculations. The comparison with Kulchitsky \& Latyshev showed the best agreement of all foils with GEANT4 and the largest difference with GEANT3, EGSnrc and PENELOPE. For the Hanson et al. gold foil comparison, again GEANT4 gave the best results, most likely due to the fact that during the development the electron scattering theory in GEANT4 was tuned to reproduce the gold data. In the beryllium foil comparisons, EGSnrc, MCNPX and PENELOPE (v. 2005) gave the best description. For the overall comparisons, EGSnrc and PENELOPE (v. 2005) were in good agreement to both the experimental data sets.

Electron scattering discussed in ICRU report 35 (1984) looks at a simplified geometry for use in estimating the width of the final profile. This simple approach to look at the scattering profile is used to determine $\overline{r^{2}}(0)$, the mean square radial spread of an electron beam at the measurement plane. This can be calculated using Equation 2.7 from the $\overline{\theta^{2}}$, mean square angular spread for four different positions: initial beam (i), primary scatterer (p), secondary scatterer (s), and drift region (v) derived from mass scattering powers given in ICRU 35. 


$$
\overline{r^{2}}(0)=\overline{r_{i}^{2}}+\left(\overline{\theta_{i}^{2}}+\overline{\theta_{p}^{2}}\right) p^{2}+\overline{\theta_{s}^{2}} s^{2}+\overline{\theta_{v}^{2}} p^{2} / 3
$$

This approximation can be used to estimate the scattering contribution from various constituents of the set-up and also the total scattering of the combination of the constituents. This simplified geometry model can be extended for additional scatterers based on the mass scattering powers and the distance to the measurement plane. The model can also be used in experimental set-up refinement by way of reducing the thickness of a scattering component or changing the gas present in the beam path.

For any measurement, one needs to have the best possible detector. The ideal detector to consider for the measurements would i) be a point detector, ii) be insensitive to other types of radiation, and iii) not be dependent on energy or dose-rate. Some possible detectors to use for this would be a point-like ion chamber, diode or 1-D detector-like arrays, or 2-D detectors like radiographic or radiochromic films. For point-like detectors, diodes and ion chambers can both have very good spatial resolution and can be useful for accurate profile measurements. Some other point-like detectors include MOSFETs (metal-oxide-semiconductor field-effect transistor), TLD (thermo-luminescent dosimeter) and OSL (Optically Stimulated Luminescence). These are not very useful for beam profile measurements since: there is no online measurement, there is no instant readout, and they can only measure at a single point before readout.

While the use of film in profile measurements might be ideal, it was not possible with no facilities for readout, analysis and calibration. It is known from other work that film precision could be a problem. For this study, both a micro-ion chamber and a diode was used for the main measurements. A comparison of three ion chambers and three diodes was also carried out. Table 2.1 outlines some of the advantages and disadvantages of four main dosimetric systems. 
Table 2.1: Main advantages and disadvantages of the four commonly used dosimetric systems. Taken from Radiation Oncology Physics: A Handbook for Teachers and Students, IAEA, 2005

\begin{tabular}{|c|c|c|}
\hline & Advantages & Disadvantages \\
\hline Ionization & Accurate and precise & Connecting cables required \\
\hline \multirow[t]{3}{*}{ Chamber } & Recommended for beam calibration & High voltage supply required \\
\hline & $\begin{array}{l}\text { Necessary corrections well under- } \\
\text { stood }\end{array}$ & $\begin{array}{l}\text { Many corrections required for high } \\
\text { energy dosimetry }\end{array}$ \\
\hline & Instant readout & \\
\hline \multirow[t]{2}{*}{ Film } & 2-D spatial resolution & $\begin{array}{l}\text { Darkroom and processing facilities } \\
\text { required }\end{array}$ \\
\hline & $\begin{array}{l}\text { Very thin: does not perturb the } \\
\text { beam }\end{array}$ & $\begin{array}{l}\text { Processing difficult to control } \\
\text { Variation between films and batches } \\
\text { Needs proper calibration against } \\
\text { ionization chamber measurements } \\
\text { Energy dependence problems } \\
\text { Cannot be used for beam calibration }\end{array}$ \\
\hline TLD & $\begin{array}{l}\text { Small in size: point dose measure- } \\
\text { ments possible } \\
\text { Many TLDs can be exposed in a } \\
\text { single exposure } \\
\text { Available in various forms } \\
\text { Some are reasonably tissue equiva- } \\
\text { lent } \\
\text { Not expensive }\end{array}$ & $\begin{array}{l}\text { Signal erased during readout } \\
\text { Easy to lose reading } \\
\text { No instant readout } \\
\text { Accurate results require care } \\
\text { Readout and calibration time con- } \\
\text { suming } \\
\text { Not recommended for beam cali- } \\
\text { bration }\end{array}$ \\
\hline Diode & $\begin{array}{l}\text { Small size } \\
\text { High sensitivity } \\
\text { Instant readout } \\
\text { No external bias voltage } \\
\text { Simple instrumentation }\end{array}$ & $\begin{array}{l}\text { Requires connecting cables } \\
\text { Variability of calibration with tem- } \\
\text { perature } \\
\text { Change in sensitivity with accumu- } \\
\text { lated dose } \\
\text { Special care needed to ensure con- } \\
\text { stancy of response } \\
\text { Cannot be used for beam calibration }\end{array}$ \\
\hline
\end{tabular}


In this work, electron scattering profile measurements were made at both $13 \mathrm{MeV}$ and $20 \mathrm{MeV}$. These energies were chosen based on several factors. Both $13 \mathrm{MeV}$ and $20 \mathrm{MeV}$ are in the therapeutic energy range and both energies bracket the Hanson et al. (1951) $15.7 \mathrm{MeV}$ energy to add to the experimental measurements of electron scattering. The variation of electron scattering powers with energy as discussed in ICRU report 35 (1984) is another factor for these two energies. It can be seen from Figure 2.1 that the change in the mass scattering power is very large at beam energy below $10 \mathrm{MeV}$. Also the experimental set-up had some limitation at low energies: the set-up would have to be adapted for the broader scattering profiles and the linac operation would not be as stable.

\subsection{Linear Accelerators}

\subsubsection{Definitions of beam characteristics}

The initial electron beam is transported through the beam pipe and impacts the exit window. This initial electron beam can be broken down into the lateral distribution, which is referred to as the beam diameter, and the angular distribution, which is referred to as the mean angular spread. Comparison between Monte Carlo calculations and experiment is complicated because the initial electron beam characteristics are not generally well known (Rogers et al., 1995). Many studies have been performed to investigate how to extract the initial beam characteristics from the linac for use in Monte Carlo calculations [Sheikh-Bagheri \& Rogers (2002), Aljarrah et al. (2006)].

\subsubsection{Rationale for use of the Vickers linear accelerator}

The main focus of this study was on thin foil electron scattering distributions. For this, the incident electron beam characteristics of the accelerator are important. Since the beam energy, diameter and mean angular spread can affect the electron scattering distribution, 
each will be briefly discussed.

Each of the three beam characteristics affects the electron scattering profiles in different ways. The beam energy affects the characteristic angle of the electron scattering profile because at high energies the distribution is more forward peaked, while at lower energies the distribution is broader with a large characteristic angle (Blais \& Podgorsak, 1992). By contrast, a variation of the incident beam diameter does not affect the characteristic angle, as shown by the study of Blais \& Podgorsak. Due to the convolution of the electron scattering kernel with the incident beam diameter, the effect of the increased diameter is smaller than the effect of the overall scattering of the components in the geometry. This is shown in Equation 2.7, compare to other components the increase of the beam diameter has a small overall effect on the end result. The mean angular spread of the beam, however, has a direct variation on the variation of the electron scattering profile. For the mean angular spread of a pencil beam, which is zero, the resulting characteristic angle will be due solely to the scattering from the foils and medium. However, in the case of an incident mean angular spread, the resulting characteristic angle will increase, as seen in Equation 2.7. Therefore, since some of the beam characteristics need to be known to study the effects of the scattering foils on the characteristic angle, the Vickers linac is an ideal machine for the investigation of electron scattering profiles as determined in the following studies.

The Vickers linear accelerator beam energy has been investigated in detail previously (MacPherson \& Ross, 1998). The energy of the beam was measured using a large magnetic spectrometer, which was built on the end of the 90-B beam pipe, the same location as used for the present study. Using a variable magnetic-field spectrometer, the energy of the incident electrons were studied. This provided the calibration of the beam energy with an uncertainty of $\pm 0.4 \%$ at the exit window. 


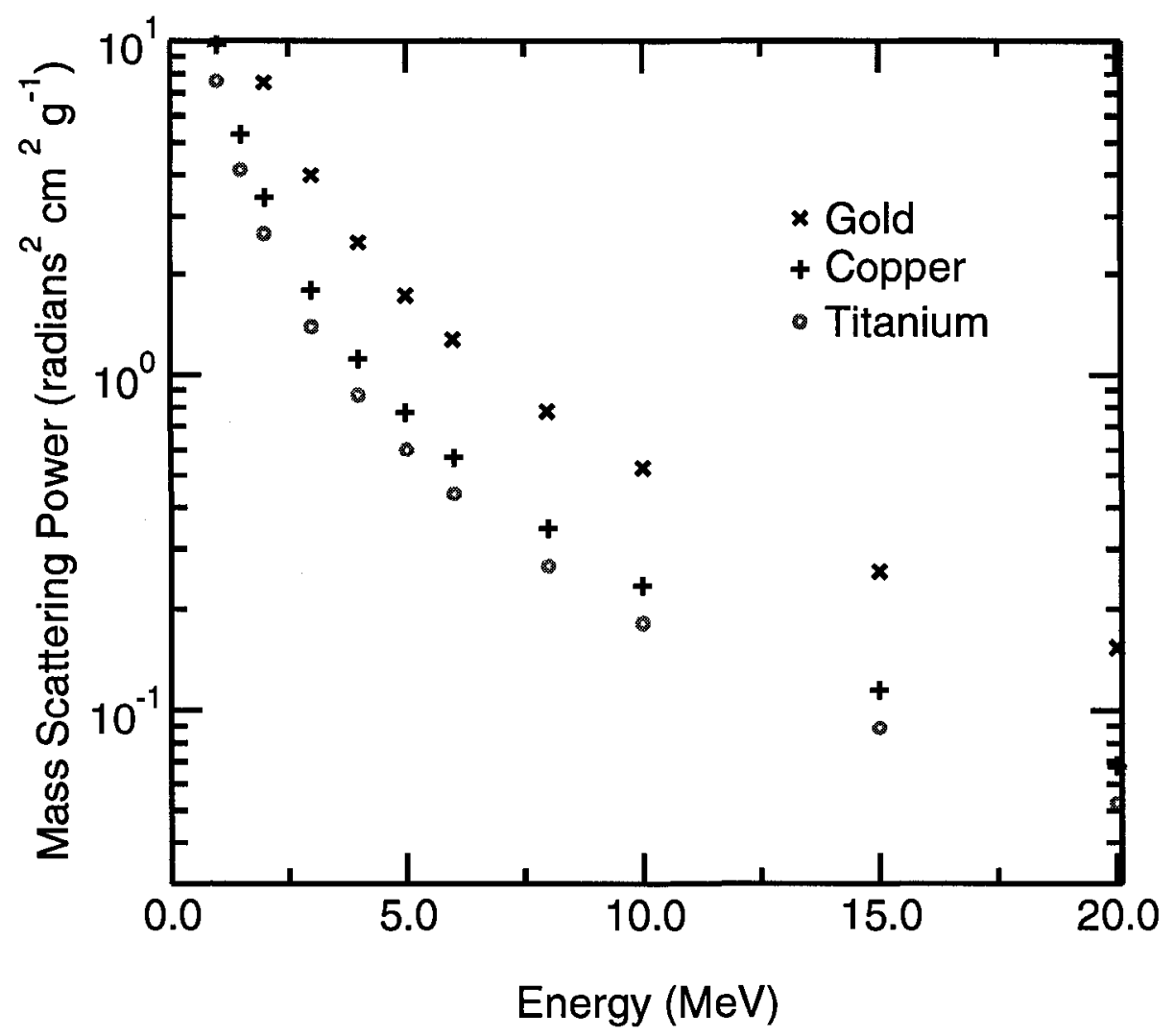

Figure 2.1: Mass scattering powers taken from ICRU Report 35 for the elements of interest. Gold $(\mathrm{Z}=79)$ was interpolated from the given data for tungsten $(\mathrm{Z}=74)$ and lead $(\mathrm{Z}=82)$ 
The beam diameter is measured using a helical wire profile monitor that rotates to provide a scan in both the vertical and horizontal directions. The monitor is located $11.0 \mathrm{~cm} \pm 0.5 \mathrm{~cm}$ from the exit window upstream in the beam pipe. The monitor scans the beam transport profile during the daily set-up and is used as a check of the accelerator prior to taking experimental data. The helical wire rotates into the path of the beam first in the horizontal direction and then in the vertical direction. When the helical wire is in the path of the beam, a current is produced in the wire. This current depends on the number of electrons passing at that location. Therefore, as the wire scans across the beam path, it is used to measure the beam diameter and spot position. The typical beam diameter was found to be $1.0 \mathrm{~mm}$. For the actual measurements, the wire profile is out of the beam.

Unlike the previous two characteristics, the beam mean angular spread has not been studied in depth. The only option for this characteristic is to place an upper limit on it determined from the geometry of the beam transport system. When the electrons exit the beam bending magnet into the $90-\mathrm{B}$ beam line, about $190 \mathrm{~cm}$ from the exit window, the steering magnets, used in directing the beam, have an inner diameter of $\sim 5 \mathrm{~cm}$. In light of the geometry of the beam transport, if the beam is as wide as the inside of the steering magnet, then it would have a radius of $\sim 2.5 \mathrm{~cm}$ and, at the exit window, has a radius of about $1.0 \mathrm{~mm}$ (as measured by the helical wire profile monitor). From these beam transport parameters, an upper limit of about $0.72^{\circ}$ was estimated for the beam mean angular spread at the exit window. The experimental location and schematic diagram can be seen in Figure 3.1 and Figure 3.2 respectively.

\subsection{Influence Quantities}

When carrying out experimental measurements, there can be many environmental quantities which cannot be controlled but which can be measured and occasionally corrected. 
These quantities will be addressed in this section. Some of the quantities for detector measurement set-ups are, but not limited to, daily temperature and pressure variations, detector leakage, ion recombination, and linac beam transport.

The temperature and pressure during the experimental runs must be monitored; changes in these parameters and can be used to correct the readings for a vented detector. Temperature and pressure corrections are routine in ion chamber measurements for comparing to standard environmental conditions. The variations in the air density are corrected to the reference conditions based on Equation 2.8.

$$
P_{T P}=\frac{273.2+T}{273.2+22.0} \times \frac{101.33}{P}
$$

where $T$ is the temperature of the ion chamber in degrees Celsius and $P$ is the pressure in kilopascals.

Another ion chamber correction is background. Background readings occur when the linac is not delivering radiation. This detector leakage is from various sources: ion chamber insulator leakage, cosmic rays and natural and man-made radiation sources. The ion chamber insulator leakage occurs when the bias voltage is applied to an ion chamber and creates a charge collection in the detector while the beam is off. If this background reading is larger than $0.2 \%$ of the peak measurement, then the profile measurements need to be corrected for this additional component.

Ion recombination is another correction quantity. In an ion chamber, the radiation beam passes through and ionizes the active medium and then the charge is collected. Under some conditions, not all of the liberated charge is collected and therefore, a correction is needed.

A bias voltage is applied across the ion chamber; the ions drift in the electric field and are collected for a measurement. Some ions will recombine during this drift period and 
the charge is lost. This can be corrected by carrying out measurements at various bias voltages. For the correction, one measures the charge collected at two voltages, $M_{\text {raw }}^{H}$ measured at $V_{H}$ (the normal operating voltage) and $M_{\text {raw }}^{L}$ measured at $V_{L}$ (a voltage at least half that of $\left.V_{H}\right)$. This correction for a pulsed beam is shown in Equation 2.9.

$$
P_{\text {ion }}\left(V_{H}\right)=\frac{1-V_{H} / V_{L}}{M_{\text {raw }}^{H} / M_{\text {raw }}^{L}-V_{H} / V_{L}}
$$

The ion recombination correction is dependent on both the bias voltage and the doserate (dose per pulse).

With a known polarity effect for ion chamber detectors, an investigation for this correction factor $\left(P_{p o l}\right)$ is required. The polarity of the bias voltage across an ion chamber can have an effect on the raw reading of the ion chamber. By taking a measurement of the set-up and only changing the polarity of the bias voltage, the correction for polarity can be easily found via:

$$
P_{p o l}=\left|\frac{\left(M_{r a w}^{+}-M_{\text {raw }}^{-}\right)}{2 M_{\text {raw }}}\right|
$$

where $M_{\text {raw }}^{+}$is the raw reading when positive charge is collected in the chamber and $M_{\text {raw }}^{-}$is the raw reading when negative charge is collected in the chamber and $M_{\text {raw }}$ is the reading corresponding to the charge collected during experimental measurements (either $M_{\text {raw }}^{+}$or $M_{\text {raw }}^{-}$) (Almond et al., 1999).

There are two aspects of the beam tuning which can also affect the measurements: the day-to-day reproducibility of the beam and the beam tuning during the measurements. The day-to-day reproducibility can be assessed by making a standard measurement each day and comparing the result to the mean of the previous measurements. The beam tuning during the measurements is controlled by a feedback system by making adjustments 
during the measurement run. The beam current is handled using a computer controlled feedback system. The beam steering and beam energy are tuned prior to measurement runs and left untouched during the measurements.

The effects of these quantities on the electron scattering profiles will be discussed in the Results section (chapter 5). 


\section{CHAPTER 3}

\section{Experimental Set-up and Measurements}

\subsection{Materials and Methods}

For this study, the Vickers 4-40 MeV linear accelerator (linac) was used. The linac is dedicated to research and is located at the National Research Council of Canada. For the experimental measurements, the $90-\mathrm{B}$ beam line was chosen because it had been studied previously (MacPherson \& Ross, 1998). The accelerator beam characteristics are well known and provide a good starting point for this study. Electron scattering profile measurements were made at both $13 \mathrm{MeV}$ and $20 \mathrm{MeV}$. The large experimental hall, which houses the linac, is an ideal location for the measurements due to the low environmental scatter (see Figure 3.1).

The various components that comprise the set-up geometry are the exit window, scattering foil, transmission monitor chamber, drift region and the detector. This can be seen in Figure 3.2. The following components were used for the final experimental set-up. The exit window was a metal alloy composed of $90 \%$ titanium, $6 \%$ aluminum and $4 \%$ vanadium with a thickness of about $43 \mu \mathrm{m}$ (mass thickness of $18.21 \mathrm{mg} / \mathrm{cm}^{2}$ ). The thin scattering foils were held in place between two aluminum plates, each having a hole of diameter $19.0 \mathrm{~mm} \pm 0.5 \mathrm{~mm}$, at a distance of $28 \mathrm{~mm} \pm 1 \mathrm{~mm}$ from the exit window. The transmission monitor chamber used to monitor the beam current was an Elekta monitor ion chamber, which was used to give the relative response to the scanning detector. One of the collecting volumes in the transmission monitor chamber was used for this relative response and another volume was used for beam control. 


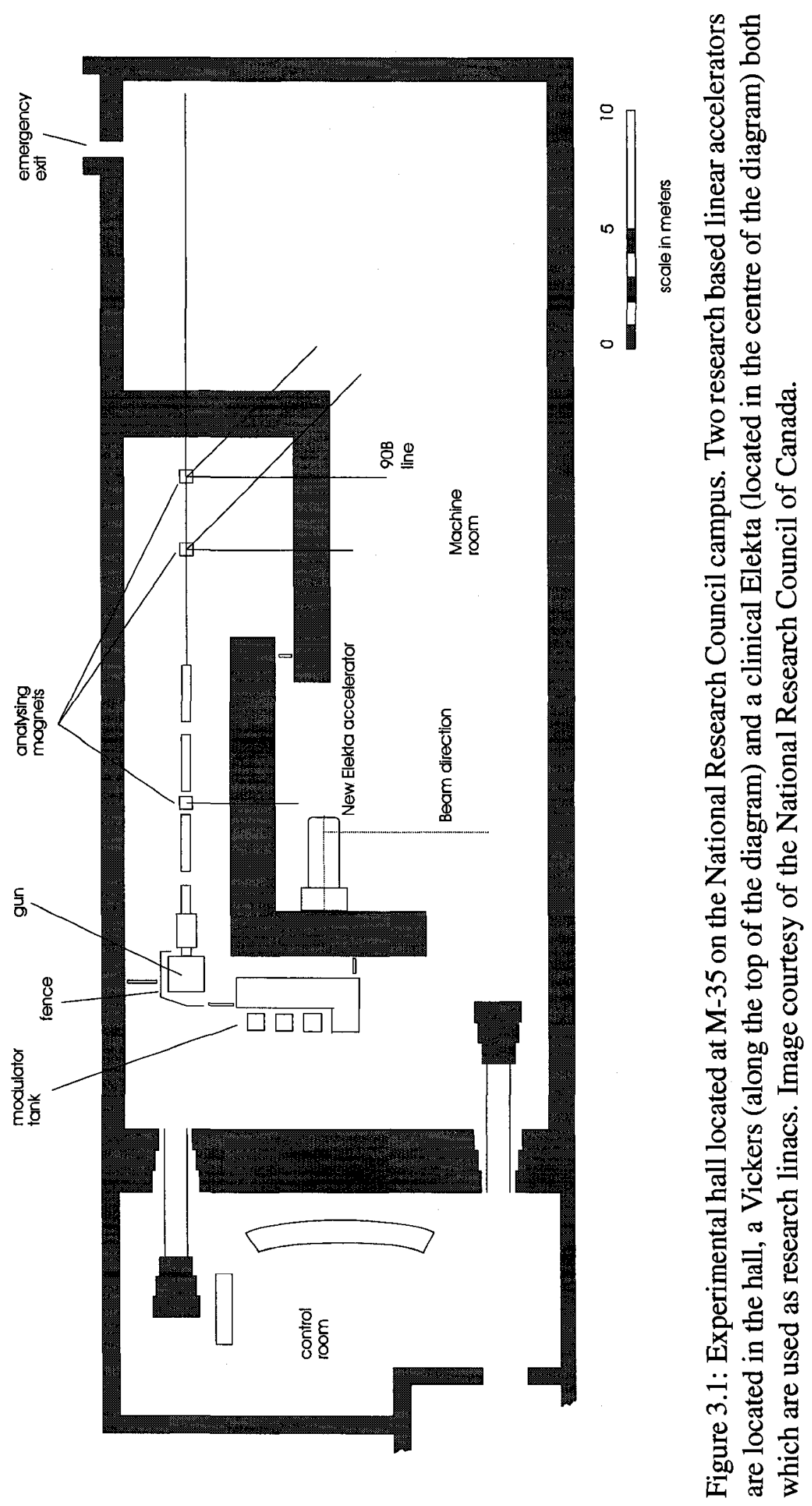




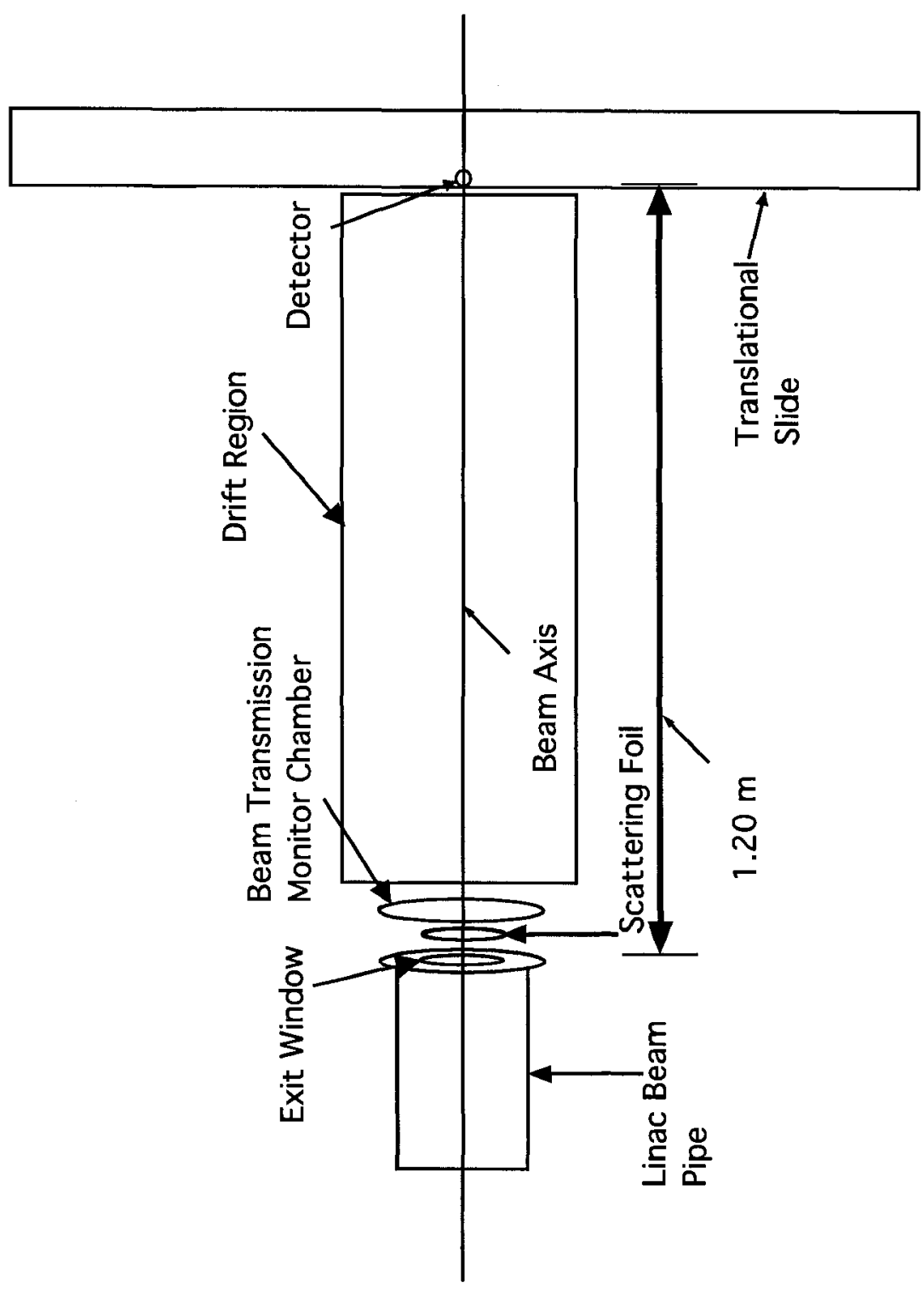

竞 
The collecting volumes were bounded by $12 \mu \mathrm{m} \pm 20 \%$ Mylar with a $12 \mu \mathrm{m}$ silk-screen layer of carbon $\left(\rho=1.13 \mathrm{~g} / \mathrm{cm}^{3}\right)$ on the Mylar (total mass thickness of $16.34 \mathrm{mg} / \mathrm{cm}^{2}$ ) (information provided by Elekta). The monitor chamber was located at $48.0 \mathrm{~mm} \pm 0.5 \mathrm{~mm}$ from the exit window.

As discussed in the literature review (Section 2.3) a vacuum chamber has been used in previous work for the drift region to minimize additional scattering. However, using a vacuum chamber with the proposed geometry set-up would have been difficult to design to be able to easily change scattering foils. As an alternative to a vacuum or air drift region, helium gas was used in the drift region due to its low scattering contribution, as can be seen in IRCU report 35 mass scattering powers (1984). The mass scattering power of helium is $30 \%$ that of air and since the physical density of helium is about $15 \%$ of that of air, the combined effect of replacing $1.1 \mathrm{~m}$ of air with helium will reduce the mass scattering contribution to about $4 \%$ of air.

The drift region was contained within an aluminized Mylar bag, which was mounted to two aluminum rings each with an inner diameter of $40 \mathrm{~cm}$. The specifications for the aluminum on the Mylar was $24 \mathrm{~nm}$ out of the total thickness of $24 \mu \mathrm{m}$. The drift region was first flushed of the ambient air with a high helium flow rate and then the flow was reduced when the percent oxygen reached $1 \%$. There was a slight positive pressure during the experimental measurements. The oxygen content was monitored using a Microelectrodes Inc. oxygen meter, model number OM-4, with a resolution of $0.1 \%$, and an MI-730 Micro-Oxygen electrode. Ambient air contains about $20 \%$ oxygen and, therefore, the concentration of helium gas can be inferred by monitoring the concentration of oxygen gas. As the concentration of oxygen decreases, as seen in Figure 3.3, then the concentration, or pressure, of the helium gas, which is flowing into the Mylar bag, must be increasing. The drift chamber was located at $65 \mathrm{~mm} \pm 2 \mathrm{~mm}$ from the exit window 
and was $111.8 \mathrm{~cm} \pm 0.5 \mathrm{~cm}$ in length when fully inflated.

While the drift region was useful for acquiring profile measurements in helium and not in air, it was also a restriction in that only profiles out to $9^{\circ}$ could be obtained.

The detector was mounted and scanned perpendicular to the beam axis (range of $50 \mathrm{~cm}$ on each side of the beam axis) on a Velmex Bislide positioning slide which was anchored to a set-up table (see Figure 3.2). The positioning slide was controlled by a VXM stepping motor via computer. This slide has a resolution of 2000 steps per $\mathrm{cm}$ and an uncertainty of $0.01 \%$. The detector was mounted at $4.0 \mathrm{~mm} \pm 3.0 \mathrm{~mm}$ from the Mylar bag at the beam axis. This meant that there was a small drift space of air between the end of the Mylar bag and the scanning detector.

Photographs of the set-up can be seen in Figures 3.4 and 3.5. In Figure 3.4, the linac can be seen on the right of the photograph with the Mylar bag in the middle of the photo. In the foreground on the left is the Exradin A16 ion chamber which is mounted on the positioning slide. On the top right hand side of the rear Mylar face is the oxygen monitor. A secondary ion chamber which was also used to monitor the beam can be seen above the Exradin A16 chamber, but it was not used in these measurements. In Figure 3.5, the linac beam pipe can be seen on the right of the photograph with the helical wire profile monitor. The exit window cannot be seen but the Elekta monitor chamber can be seen in the middle of the photographs as the black disk. The Mylar bag can be seen on the left of the photograph positioned as close as possible to the Elekta chamber. 


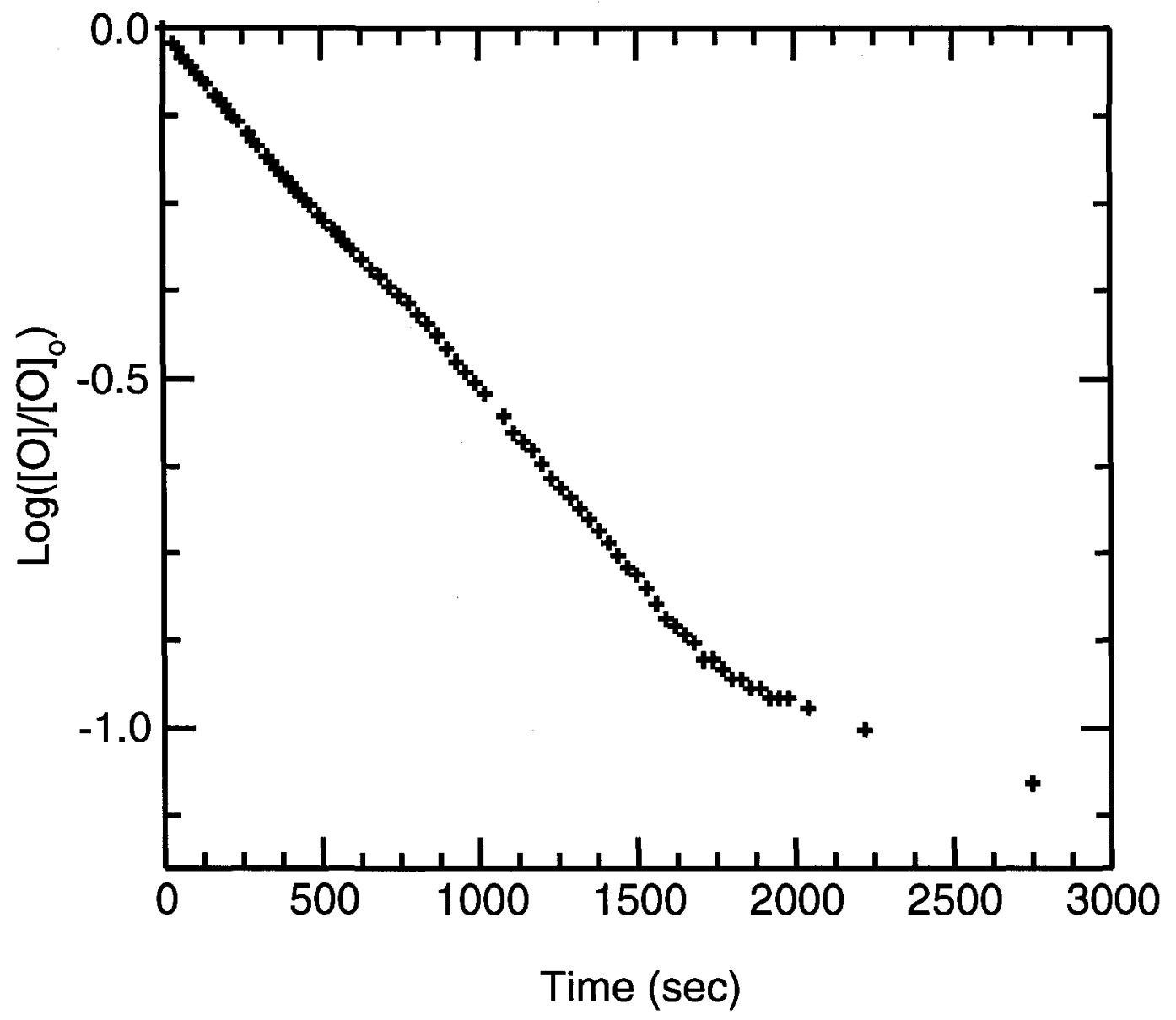

Figure 3.3: The relative concentration of oxygen during the process of filling of the aluminized Mylar bag. When the percent oxygen reached $1 \%$, the gas flow was reduced as shown by leveling off of the curve at approximately 2000 seconds. Experimental measurements were performed when the oxygen level was below $1 \%$. 


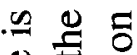
造造 छ 迹

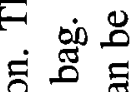
类 倦变苞 을 . of 就 음 要要 항 에

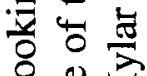
을

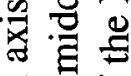
대을 ญ $0 \rightleftharpoons$ 层 政 西 통

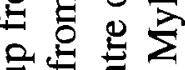
年 列. 这 跣 8 过 달 品寻

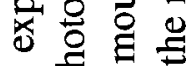
들 등 广。 3 แ - 矛至需 $\varangle$ 昰 ن. 列 政 知被。 
을

뭉

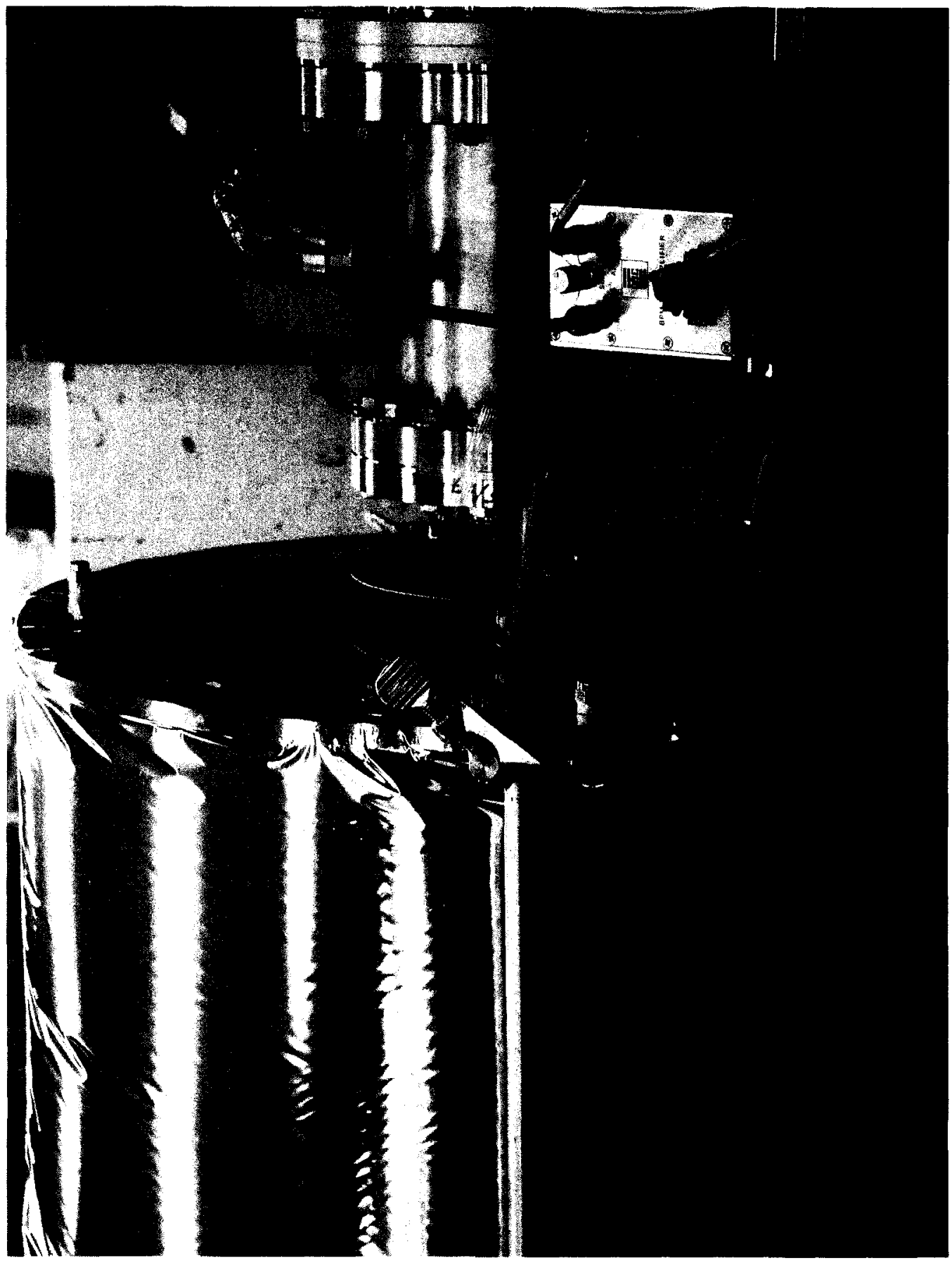

3

导

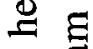

$\stackrel{乛}{ \pm}$

홇

84

줄 응

苞

- $ᄋ$

壳

옹

음

온

넝 氕

ษ

- 홍

등 홍

'

焉

ธี $\frac{0}{0}$

욕욜

它

उ $\frac{0}{0}$

芯 哥嗅

: :

贾

ชั

올

渮

근 응

.

온

요웅

《 0

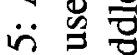

ल

는

कo

江 $\Xi$ I 


\subsection{Initial Measurements}

This study is to build on similar measurements in the area of electron scattering profiles as described in the literature review. As with most experimental measurements, the set-up was achieved iteratively after initial investigations. The set-up components were assessed for their contribution to the electron scattering profiles. The final selection of the monitor chamber was chosen to minimize its scattering contribution. Three monitor chambers were studied. The first was a Mevex chamber, with a total mass thickness of $21.64 \mathrm{mg} / \mathrm{cm}^{2}$, constructed with six layers of aluminum-coated Kapton and was previously studied on the Vickers linac (Sheikh-Bagheri et al., 2000). An in-house designed transmission chamber, with an aluminum-covered Mylar of $6 \mu \mathrm{m}$ protective outer layer and $200 \mathrm{~nm}$ gold on $125 \mu \mathrm{m}$ on Kapton collecting volume with a mass thickness of $37.98 \mathrm{mg} / \mathrm{cm}^{2}$, was also considered as a transmission monitor chamber. The Elekta chamber, taken from an Elekta Precise ${ }^{\mathrm{TM}}$ clinical accelerator (circa 2002-2005), described above in Section 3.1, had a mass thickness of $16.34 \mathrm{mg} / \mathrm{cm}^{2}$. While the Elekta chamber and Mevex chamber scans were similar, the Elekta chamber was chosen for the experimental measurements. The Elekta chamber design included three pancake-like layered charge collecting volumes on axis. The beam passes through the three collecting volumes, enabling a feedback to the linac control system and for relative response measurements. This design is unlike the Mevex chamber where the collecting volumes are two layers of two off-axis semicircles, which has a spot in the centre of the beam axis which does collect charge.

\subsection{Scattering Foils}

The electron scattering distributions from three materials, a titanium alloy, copper and gold, were investigated. In target scattering experiments, the most important parameter is 
the mass thickness of the targets. The mass thickness of a foil is calculated by one of two methods: using the thickness and the known density, and using the the area and the mass. The first method requires that the density of the foil material be known. However, one of our foils is a titanium alloy whose density is not accurately known. For this reason, the second method was adopted. The measurement of the area was performed using a digital camera mounted on a tripod. The foils were placed, along with a ruler, on a black platform. A picture was taken and then the area was extracted using software, based on the pixel/mm ratio. Finally, the mass was measured and the mass thickness of the foil calculated.

The measured mass thickness for each foil is shown in Table 3.1. For the gold and titanium alloy series, multiple foils were cut from a single sheet, for a series of profile measurements. The copper series used foils which were cut from separate sheets. The profiles were measured for 1 to 5 samples for each of the foils. The large sheet was assumed to have a uniform thickness and the mass thickness was expected to be a multiple of a single foil.

Table 3.1: Measured mass thickness of the scattering foils used in the experimental measurements. For each foil a series of measurements was performed using multiple foils. Two different foil thicknesses were used for the gold series in the profile measurements.

\begin{tabular}{|c|c|c|c|c|}
\hline Foil & $\mathrm{Z}$ & Tabulated density $\left(\mathrm{g} / \mathrm{cm}^{3}\right)$ & \multicolumn{2}{|c|}{ Mass Thickness $\left(\mathrm{mg} / \mathrm{cm}^{2}\right)$} \\
\cline { 4 - 5 } & & & Thin & Thick \\
\hline Titanium Alloy & $21.68^{a}$ & 4.42 & 18.2 & - \\
Copper & 29 & 8.92 & $43.5^{b}$ & - \\
Gold & 79 & 19.30 & 31.0 & 54.9 \\
\hline
\end{tabular}

${ }^{a}$ Effective Atomic Number

${ }^{b}$ Average Mass Thickness 


\subsection{Data Acquisition}

The measurements were performed using a detector mounted on a computer-driven translational slide and the detector was scanned into position. Once the detector was in position, a signal was sent from the computer to the two Keithey 35617 electrometers, each to trigger the transmission monitor chamber and the scanning chamber, to begin charge collection. The two electrometers were triggered simultaneously using the GPIB Group Execute Trigger (GET) function. This function stores the GPIB addresses in an array and then an action can be sent to the group for simultaneous execution. This method of operation was used to reduce the time delay between the measurements of the two detectors to sample the same beam. The charge was collected at each measurement point for an integration time of 5.0 seconds. During the overall measurement, the detector scans from one side of the profile to the other side and back, stopping at predetermined points.

The transmission monitor chamber was used to normalize for the incident beam current variations that might occur over the course of the measurement. The ionization current (normalized to the average), which can be seen in Figure 3.6, shows the standard deviation of the incident beam current is $\pm 2.2 \%$ over the $10-15$ minute measurement run. However, these variations in the beam current do not affect the profile because the response of the scanning detector is relative to the transmission monitor chamber.

\subsection{Detectors}

For the electron scattering profile measurements, various ion chambers and diodes were investigated, as seen in Table 3.2. These detectors were compared using the most simple set-up: the thin exit window, the Elekta monitor chamber, no scatter foil and a helium drift region. The ion chambers and diodes were chosen to get a range of sizes which are commercially available. Ion chambers have a density of charge carriers of approximately 


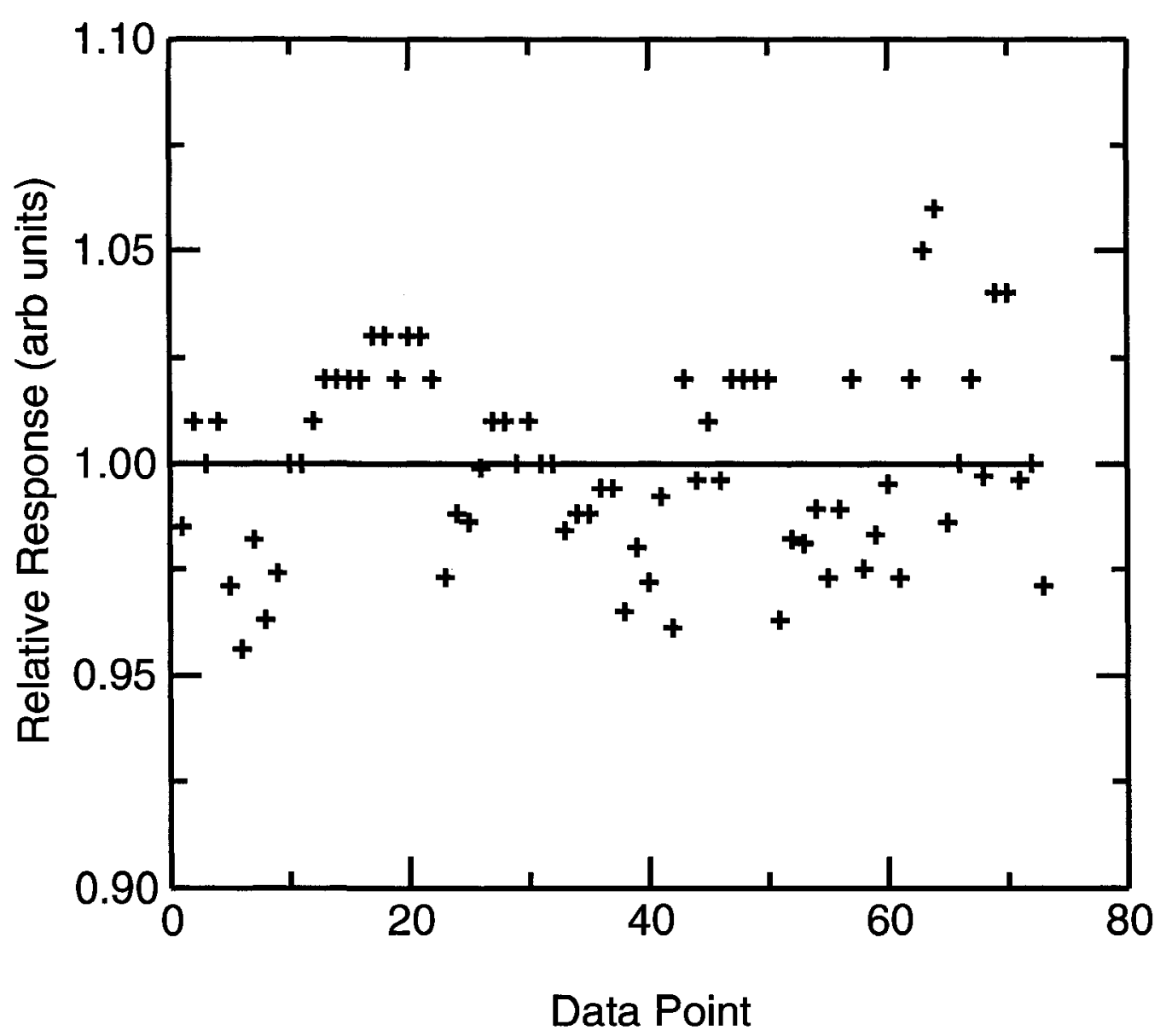

Figure 3.6: The ionization current (normalized to the average) of the incident beam over the course of a typical run as measured by the Elekta transmission monitor chamber. 
$1 / 2000$ that of diodes. This is due to the differences in the mass density. For the detector measurements, the ion chamber will be measuring dose-to-air and the diode will be measuring dose-to-silicon. 


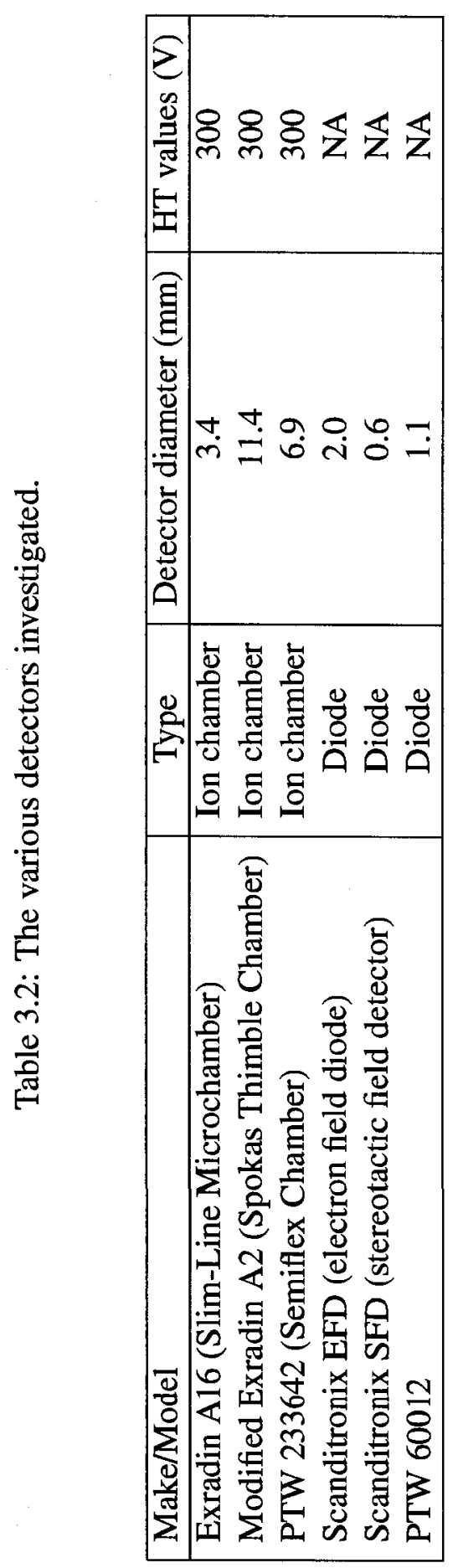




\section{CHAPTER 4}

\section{Monte Carlo Simulations}

\subsection{Monte Carlo Codes}

For this study, EGSnrc (Kawrakow \& Rogers, 2000) was used because it has been previously used for studying Monte Carlo radiation transport comparisons of the Vickers linac (Sheikh-Bagheri et al., 2000). EGSnrc (Electron Gamma Shower NRC) is a Monte Carlo package used to simulate coupled electron photon radiation transport. The current energy range for application is considered to be from $1 \mathrm{keV}-10 \mathrm{GeV}$. The overlying user codes BEAMnrc, DOSRZnrc and FLURZnrc were used for the simulations. For all Monte Carlo simulations, the PEGS (Preprocessor for EGS) data set was the provided default data set, 700ICRU.

\subsection{BEAMnrc}

BEAMnrc (Rogers et al., 2005b), which is an extension of BEAM (Rogers et al., 1995) is used for modeling radiation sources used in radiation transport and is based on the framework of the EGSnrc Monte Carlo system. BEAMnrc can simulate external beam treatment linacs in great detail and has been shown to give good agreement with measurements (e.g. Tonkopi et al., 2005). In the BEAMnrc system, there is user specified information to the main input file: the input radiation source, the parts of the treatment head, if any, and the modes of output for the particles at the end of the geometry. Many different types of sources can be modeled in BEAMnrc, such as a point source, a pencil beam or a broad beam. The component modules, which are different materials in various 
configurations, are located after the incident electron beam. Some examples of component modules are SLABS, CONESTAK, JAWS, MIRROR, all of which can make up the simulation geometry of a clinical linac. SLABS is a component module used for multiple square slabs of arbitrary thickness and medium that are perpendicular to the $\mathrm{z}$-axis of the beam. CONESTAK is used to simulate truncated cones, a circular layer of arbitrary thickness with a user-defined inner and outer radius at both the top and bottom. JAWS, which is a square layer of an arbitrary thickness, is used to simulate sets of beam-defining jaws that are used in collimating the beam. JAWS can be set open or closed in either the X or $\mathrm{Y}$ direction and can be set at an angle from the z-axis. MIRROR simulates the mirror used in the linac treatment head and is positioned at an arbitrary angle, with respect to the $\mathrm{z}$-axis and set at an arbitrary thickness. Of these modules, only SLABS and CONESTAK are relevant for this investigation.

In the BEAMnrc input file, the main components of the set-up were modeled: exit window, scattering foil, monitor chamber, Mylar walls of the drift region, and the drift region itself. In the current study, the input source that most resembles the Vickers linac was source ISOURC $=19$, "a circular beam with 2-D Gaussian X-Y distribution, parallel or with radial divergence" (Rogers et al., 2005b, p. 72) (see Figure 4.1). Using this source, one can vary the parameters that are relevant to the experimental measurements, such as the incident beam diameter and incident mean angular spread. The BEAMnrc environment was first filled with air to simulate the real environmental conditions instead of the default vacuum, and all the component modules were put into place. The input geometry was broken down into two component modules: SLABS and CONESTAK. These were chosen due to the simple experimental set-up geometry of this study.

The component module SLABS was used for the exit window, which was located at $0.0 \mathrm{~cm}$ from the beginning of the geometry and the scattering foil which was placed at 


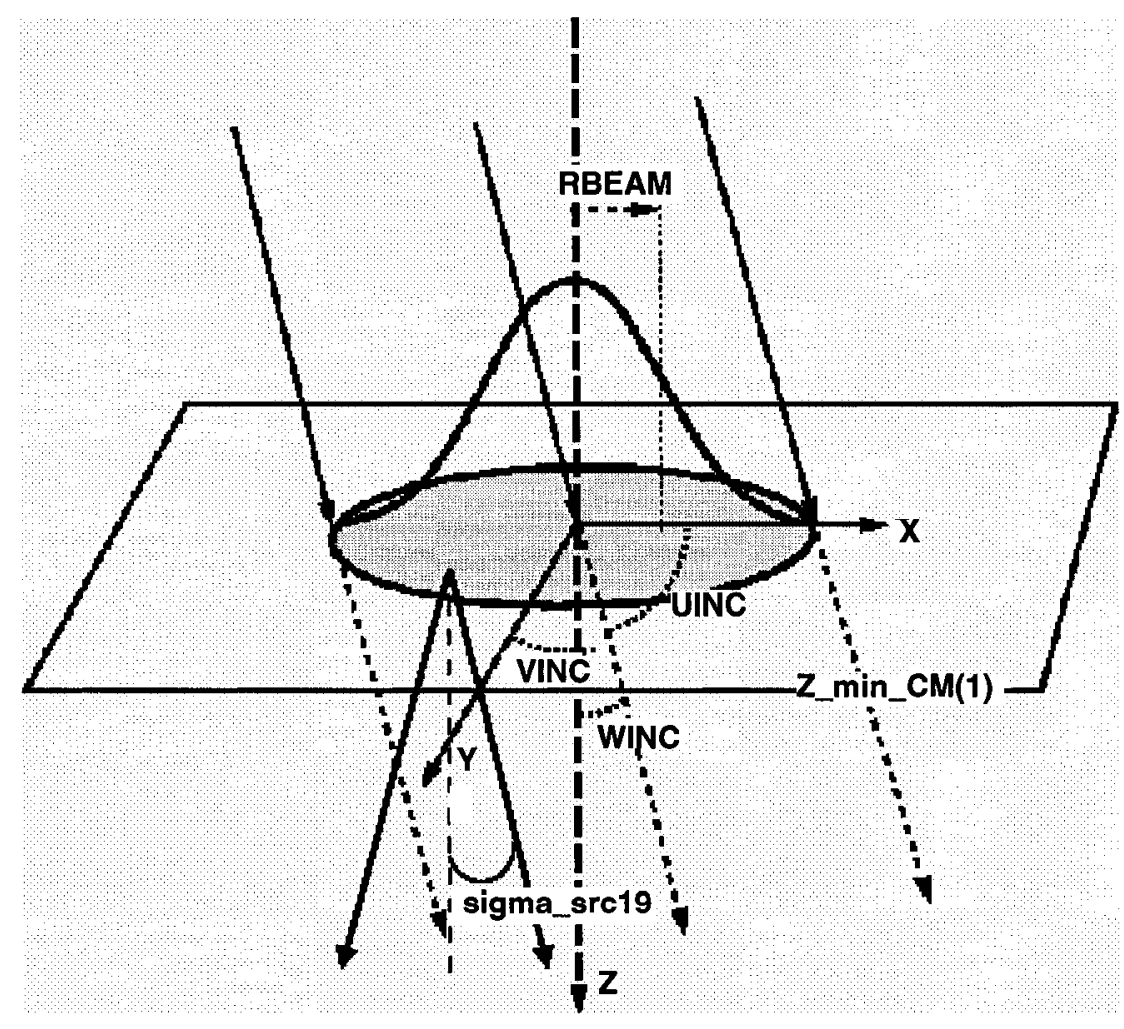

Figure 4.1: "ISOURC=19, Circular Beam with 2-D Gaussian X-Y Distribution, Parallel or with Radial Divergence, RBEAM = the standard deviation of the X-Y Gaussian distribution, or the negative full width half max (-FWHM) of the X-Y Gaussian distribution. The beam can either have mean angular spread of sigma_src19 or the incident beam cosines specified by UINC, VINC, WINC for the X, Y, Z directions" (Rogers et al., 2005b). The diameter of the beam can be denoted by the standard deviation of a Gaussian distribution by using a positive number or the FWHM of the Gaussian distribution by using a negative number. The mean angular spread of the incident electrons is based on a Gaussian distribution of standard deviation sigma_src 19. 
$2.8 \mathrm{~cm}$ from the beginning of the geometry. All distances were set from the beginning of the geometry. These SLABS were kept at the default $25.0 \mathrm{~cm}$ by $25.0 \mathrm{~cm}$ square, simply because the electron beam distribution, at a distance of $2.8 \mathrm{~cm}$, was within the boundaries of the experimental foils, and therefore, there was no need to model the true outer dimensions and shape of the foils. The monitor chamber was set at a distance of $4.85 \mathrm{~cm}$, and used the CONESTAK component module as it contained many layers with inner and outer radii. The monitor chamber was modeled as six layers of $24.0 \mu \mathrm{m}$ Carbon Graphite composite (based on estimates of the technical specifications) separated by air. This approximation of the monitor chamber was due to the fact that the true technical specifications of the Elekta monitor chamber were not available until late into the project. The Mylar bag and drift region were modeled using SLABS. The Mylar of the bag was $24.0 \mu \mathrm{m}$ thick, ignoring the aluminum, and the drift region was $\sim 115 \mathrm{~cm}$.

During the simulation, there are different parameters required. Some examples of these include, but are not limited to, the cut-off energy of an electron and photon and the bremsstrahlung cross-section. For the Monte Carlo calculations, the default transport parameters were used and the following input parameters were specified:

\begin{tabular}{|c|c|}
\hline Input & Value \\
\hline Source & $19($ see Figure 4.1 \\
Input particles & $20.0 \mathrm{MeV}(13 \mathrm{MeV})$ electrons \\
RBEAM & $2.0 \mathrm{~mm}$ \\
Histories & $1.0 \cdot 10^{7}$ \\
Bremsstrahlung cross-section & $\mathrm{BH}$ (Bethe-Heitler) \\
\hline
\end{tabular}

Table 4.1: Input parameters used in all BEAMnrc calculations

BEAMnrc was used as a shared library with DOSRZnrc as this would reduce hard drive space and also speed up the time required for the simulation. For a calculation us- 
ing a shared library, the particles start from the beginning of the BEAMnrc input source simulation and are tracked though the geometry until the end of the BEAMnrc geometry. When the particles reach the boundary, they are then tracked within the DOSRZnrc geometry.

\subsection{Other EGSnrc Tools}

Both DOSRZnrc and FLURZnrc were used for Monte Carlo calculations of the experimental set-up. DOSRZnrc was used for the primary comparison and investigations while FLURZnrc was used for a secondary investigation. DOSRZnrc is a user code based on the EGSnrc physics package used to score dose in any material in a right cylindrical configuration (Kawrakow \& Rogers, 2000). DOSRZnrc is like all other EGSnrc codes where input files are used for the geometry and parameters of the simulations. For the simulations with DOSRZnrc the medium used to score dose and the geometry are in an input file along with the data set for the medium.

For this study, DOSRZnrc was used as opposed to the planar configuration of DOSXYZnrc due to the symmetry of the set-up geometry and measurements. DOSRZnrc was used to score dose with air rings $2.0 \mathrm{~mm}$ in depth. The thickness of the scoring plane was chosen based the average of the two main detectors. BEAMnrc was used as a shared library with DOSRZnrc, by way of input source type number 23 "full BEAM simulation, incident from any angle/position" (Rogers et al., 2005a, p. 28)

FLURZnrc is another tool based on the EGSnrc package. It was used to study the energy fluence and particle fluence from input sources. The package scores fluence in any material in a right cylindrical configuration (Kawrakow \& Rogers, 2000). FLURZnrc was only used in the investigations of the secondary radiation effects. For this investigation the BEAMnrc source file of the thickest gold foil $\left(218.7 \mathrm{mg} / \mathrm{cm}^{2}\right)$ was used to investigate 
the energy fluence for both electrons and photons at the measurement plane. Again this was scored in air rings with a depth of $2.0 \mathrm{~mm}$ with energy bin widths of $200 \mathrm{keV}$ each. Like DOSRZnrc, the FLURZnrc code also used BEAMnrc as a shared library for the source of particles, again using input source type number 23.

The Monte Carlo transport parameters used in each of the tools were the default values, except for the following:

\begin{tabular}{|c|c|}
\hline Input & Value \\
\hline & \\
Source & 23 \\
PEGS & $700 \mathrm{ICRU}$ \\
Histories & $1.0 \cdot 10^{7}$ \\
\hline
\end{tabular}

(a) DOSRZnrc input parameters

\begin{tabular}{|c|c|}
\hline Input & Value \\
\hline IPRIMARY & Total fluence \\
Source & 23 \\
PEGS & $700 \mathrm{ICRU}$ \\
Histories & $1.0 \cdot 10^{8}$ \\
\hline
\end{tabular}

(b) FLURZnrc input parameters

Table 4.2: Input parameters used in the calculations for (a) DOSRZnrc and (b) FLURZnrc. 


\section{CHAPTER 5}

\section{Results and Discussion}

\subsection{Analysis of the Data}

Figure 5.1 shows a typical measurement for an experimental set-up: the exit window, a copper scattering foil, the transmission monitor chamber and a helium drift region. According to the ICRU report 35 (1984), the electron scattering distributions are not Gaussian. From electron scattering theory, the profile is a combination of a Gaussian based on the small angle scattering and a non-Gaussian due to the large angle scattering in the tails. For this investigation, the application of a single parameter to describe the scattering profile is very useful for making comparison. The characteristic angle $\left(\theta_{c}\right)$ of a Gaussian has been used previously to fit these electron scattering profiles [Hanson et al. (1951), Blais \& Podgorsak (1992), and Kulchitsky \& Latyshev (1942)]. Therefore, this technique will also be used here.

The main difference between the Monte Carlo and experimental results are the presentation of the calculated and measured outcomes. Whereas the experimental measurement is a one dimensional scan across the scattered beam, the Monte Carlo calculation is a series of concentric rings in which the dose is deposited within each ring. Using the process described in Section 5.2, the experimental measurements are easily transformed for comparison.

For a Gaussian:

$$
R(\theta)=r_{o}+A e^{\left(\frac{-(\theta-\mu)^{2}}{\theta_{c}^{2}}\right)}
$$

where $\mathrm{R}(\theta)$ is the relative response at $\theta, \theta$ is the off-axis angle, $r_{o}$ is the measurement 


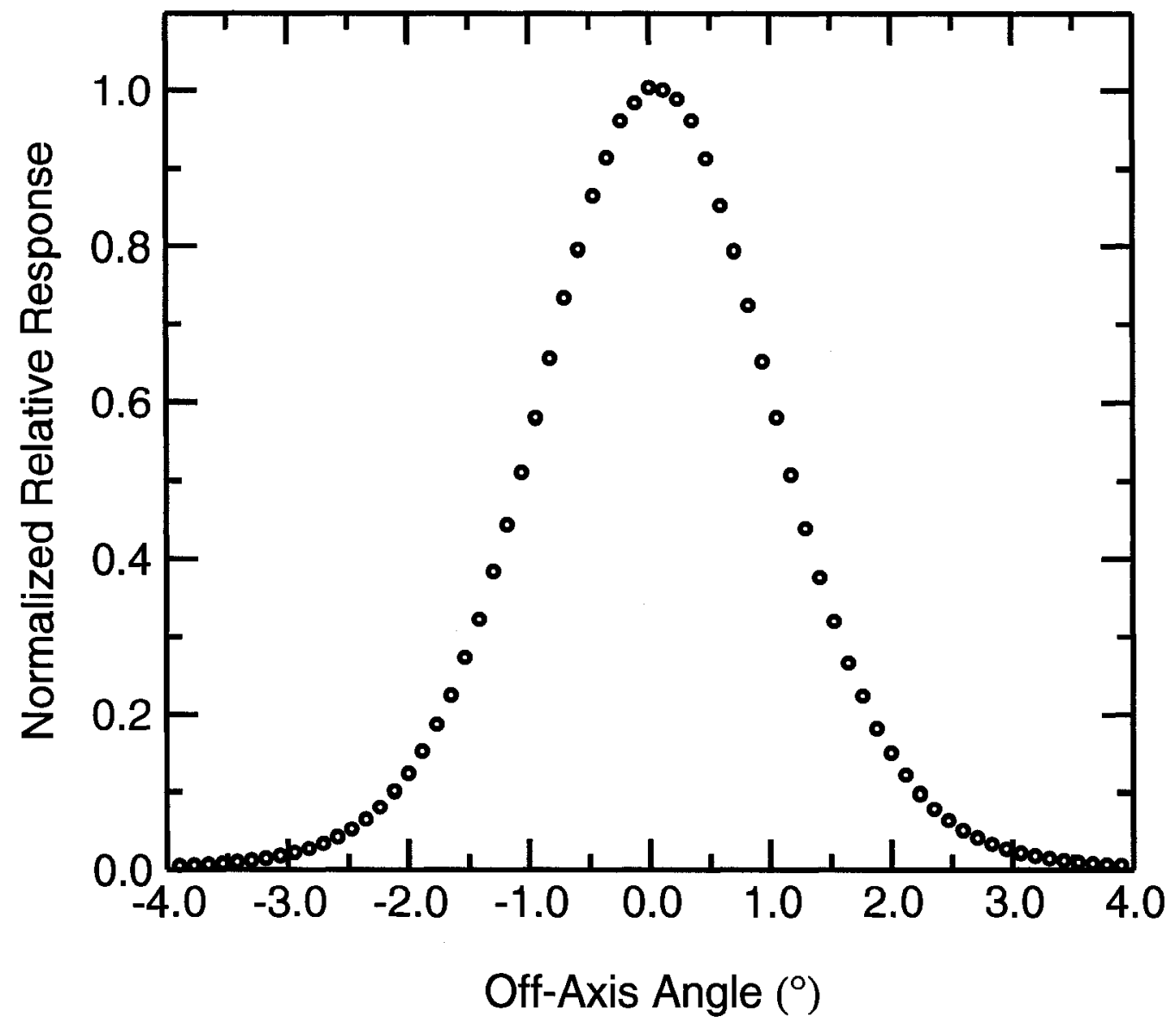

Figure 5.1: An example of a typical experimental measurement. Profile from a copper scattering foil $\left(43.63 \mathrm{mg} / \mathrm{cm}^{2}\right)$ at $20 \mathrm{MeV}$. 
background, $\mathrm{A}$ is the height of the Gaussian peak, $\mu$ is the location of the central peak of the distribution, and $\theta_{c}$ is the characteristic angle, the angle at which the intensity falls to $1 / \mathrm{e}$ of the peak intensity.

\subsection{How the Characteristic Angle was Extracted}

The steps outlined below were taken to extract the characteristic angle. The relative response of the scanning detector was calculated by taking the ratio of the charge collected in the scanning detector to the charge collected in the monitor chamber. This is the electron scattering profile. For the analysis of the experimental measurements, the profile was mirrored and then both sides of the mirrored data were fitted with a single fifth order polynomial. The fifth order polynomial was used to determine the shift required to minimize the difference between the origin of the detector and the true centre of the profile. This shift was adjusted to the sub-millimeter level iteratively to minimize the difference. Due to this shift of the central peak of the profile, the value of $\mu$ was taken to be zero. The zeroth order coefficient was used to normalize the relative response profile. The absolute value of the corrected off-axis distance was converted to the off-axis angle based on the distance from the exit window to the measurement plane. The background and detector leakage of the profile was measured to be zero and thus $r_{o}=0$.

The modified data for the natural logarithm of the normalized relative response versus the square of the off-axis angle was then plotted, this can be seen in Figure 5.2. In the ideal Gaussian distribution case, this would result in a straight line as seen in Equation 5.2 and could be used to extract the characteristic angle of the profile.

$$
\ln \frac{R(\theta)}{A}=\left(\frac{-\theta^{2}}{\theta_{c}^{2}}\right) .
$$

The slope of this line can be used to extract the characteristic angle, as seen in Equa- 
tion 5.3.

$$
\theta_{c}=\left(\frac{-1}{\sqrt{m}}\right)
$$

where $m$ is the slope of the plot of natural logarithm of the normalized relative response $\left(\ln \frac{R(\theta)}{A}\right)$ versus the square of the off-axis angle $\left(\theta^{2}\right)$. For the case in this study, the peak of the distribution was taken to be Gaussian and preceding procedure was used. The tails of the profile did not follow a Gaussian therefore, they were not used in the extraction of the characteristic angle. This will be addressed in Section 5.2.2. A hands-on process for extracting the characteristic angle was used as opposed to using a 'black box' data analysis package.

The technique for extraction of the characteristic angle in the Monte Carlo calculations was similar but had one significant difference. Since the Monte Carlo calculations were circularly symmetric and the output was dose per radial bin, a centering procedure was not required. The off-axis distance was converted to the off-axis angle as before. The natural logarithm of the normalized relative response and the square of the off-axis angle were again used to obtain the characteristic angle. This procedure to extract the characteristic angle for both the experimental measurements and the Monte Carlo calculations was taken over the same range of off-axis angle to reduce bias (see Section 5.2.2).

\subsubsection{Peak of the distribution}

The position of the beam needs to be well known since the profile is non-Gaussian. The characteristic angle of a Gaussian distribution is the same taken over any portion of the profile, but for a non-Gaussian distribution an off-centre profile will affect the characteristic angle. The positional laser (parallel to the beam axis) points at the centre of the exit window and so was used to align the detector with the origin for each of the scans. The 


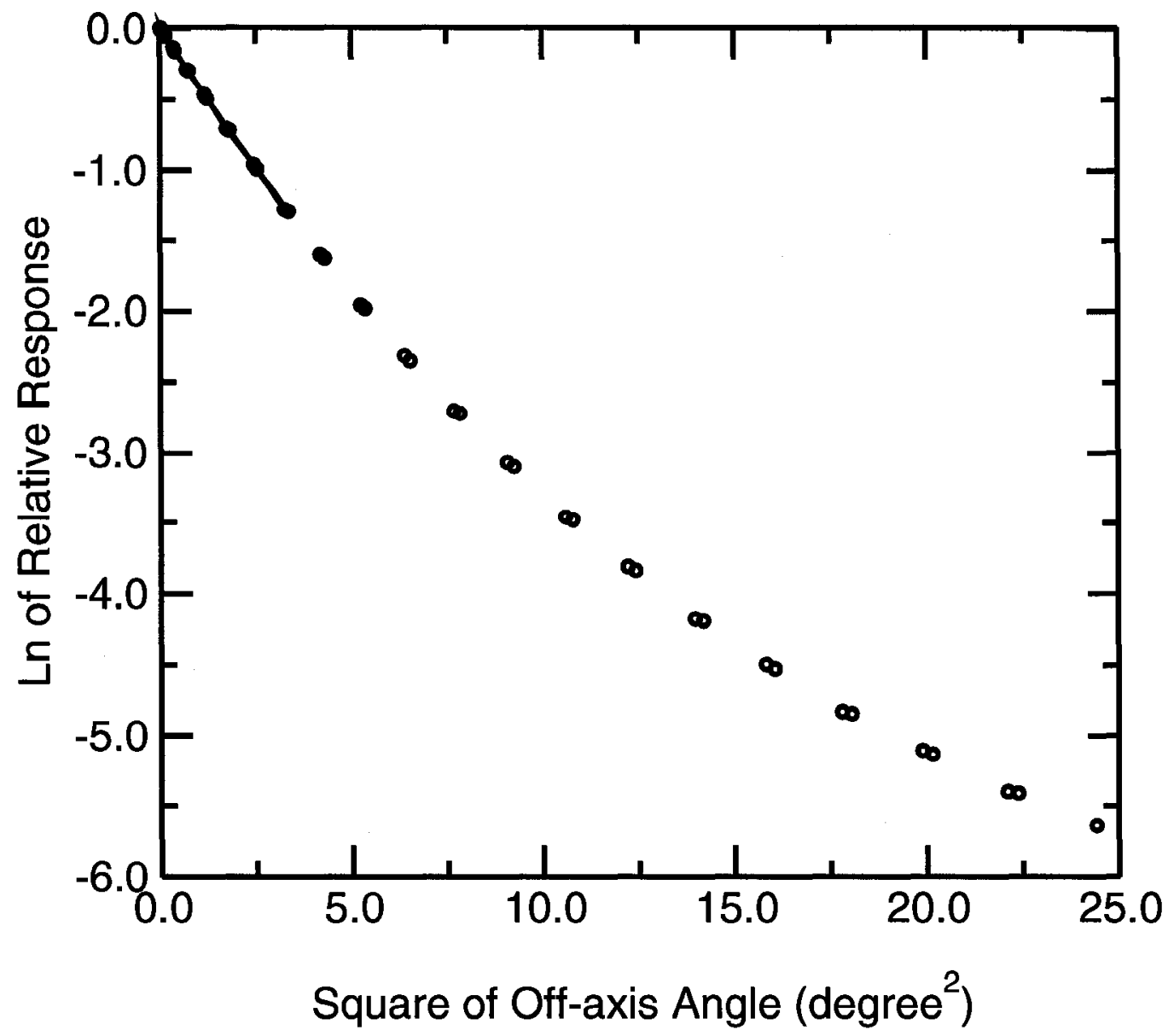

Figure 5.2: Typical plot for the extraction of the characteristic angle for a titanium alloy $\left(18.21 \mathrm{mg} / \mathrm{cm}^{2}\right)$ scattering set-up of $20 \mathrm{MeV}$. 
laser spot was approximately $1.0 \mathrm{~mm}$ wide at the scanning plane. During the fitting of the experimental results, there was a mirror and shift routine which left the characteristic angle insensitive to misalignment in the scanning slice (the horizontal axis). However, a misalignment in the vertical axis can also occur during the initial positioning of the detector. A theoretical investigation showed that a detector off-set $5.0 \mathrm{~mm}$ too high or too low from the absolute beam peak, leads to a $0.5 \%$ increase in the characteristic angle for the $20 \mathrm{MeV}$ window only case. Likewise, a $1.0 \mathrm{~cm}$ off-set leads to a $1.0 \%$ increase in characteristic angle. This was confirmed with experimental measurements. The simplest scattering set-up is the narrowest profile. Therefore, a positional misalignment has the largest effect on the characteristic angle. However with the detector positioning better than $3.0 \mathrm{~mm}$ (worst case), the characteristic angle can be extracted with an uncertainty of $0.3 \%$.

\subsubsection{Sensitivity of characteristic angle over data range}

Since the distribution is not totally Gaussian, varying the fitting range changes the resulting characteristic angle. While most of the measured profiles did include the tails, these were not needed in the extraction of the characteristic angle. The part of the profile used was based on the line of best fit for the plot of logarithm of the normalized relative response versus the square of the off-axis angle. This can be seen in Figure 5.2. In this example, the straight line portion of the square of the off-axis angle from 0 to 3.3 shows where the characteristic angle was extracted. The value of natural logarithm of the normalized relative response greater than -1 was used to extract the characteristic angle $\left[\left(\ln \frac{R(\theta)}{A}\right) \geq-1\right]$. Beyond the characteristic angle the line starts to curve significantly which means this part of the profile is not Gaussian. Changing the fitting range around this point by $10 \%$ leads to a variation up to $3 \%$ for the absolute value of the charac- 
teristic angle. However, when comparing the characteristic angle over the same range between Monte Carlo calculations and experimental measurement or from run to run this sensitivity drops out.

\subsection{Beam Characteristics}

Since the beam characteristics are important for the electron scattering profile, the variation in each characteristic needs to be addressed along with the corrections needed for the measurements. Although the effect of the beam diameter of the characteristic angle has been studied previously with claims that the initial beam diameter does not affect the characteristic angle (Blais \& Podgorsak, 1992), an investigation of the beam diameter was carried out to verify this result. The initial beam diameter was measured using the helical profile monitor as described in Section 2.4.2. Prior to an electron scattering profile measurement, the wire was scanned in the vertical plane and the horizontal plane. The profile is shown in Figure 5.3. While it is noisy, the beam profile was used to extract the characteristic width of the initial beam. The beam diameter was extracted from these profiles using the same technique as that for the characteristic angle of the final measurements as discussed in Section 5.2. The characteristic width of the initial beam diameter was measured to be $0.70 \mathrm{~mm}$ (FWHM of $1.17 \mathrm{~mm}$ ) in the vertical direction and $0.50 \mathrm{~mm}$ (FWHM of $0.83 \mathrm{~mm})$ in the horizontal direction $\left(x_{\mathrm{FWHM}}=2 \sqrt{\ln 2} \cdot x_{c}\right)$. From these results, the initial beam is considered an elliptical Gaussian distribution which is not surprising as the optics of beam transport usually focus in one plane and defocus in the other (Fernow, 1986).

A Monte Carlo investigation of the effect of the initial beam diameter shows a very small variation in the characteristic angle of the final profile. In the most simple set-up, the initial beam full-width half-maximum was varied from $1.0 \mathrm{~mm}$ to $4.0 \mathrm{~mm}$, which 


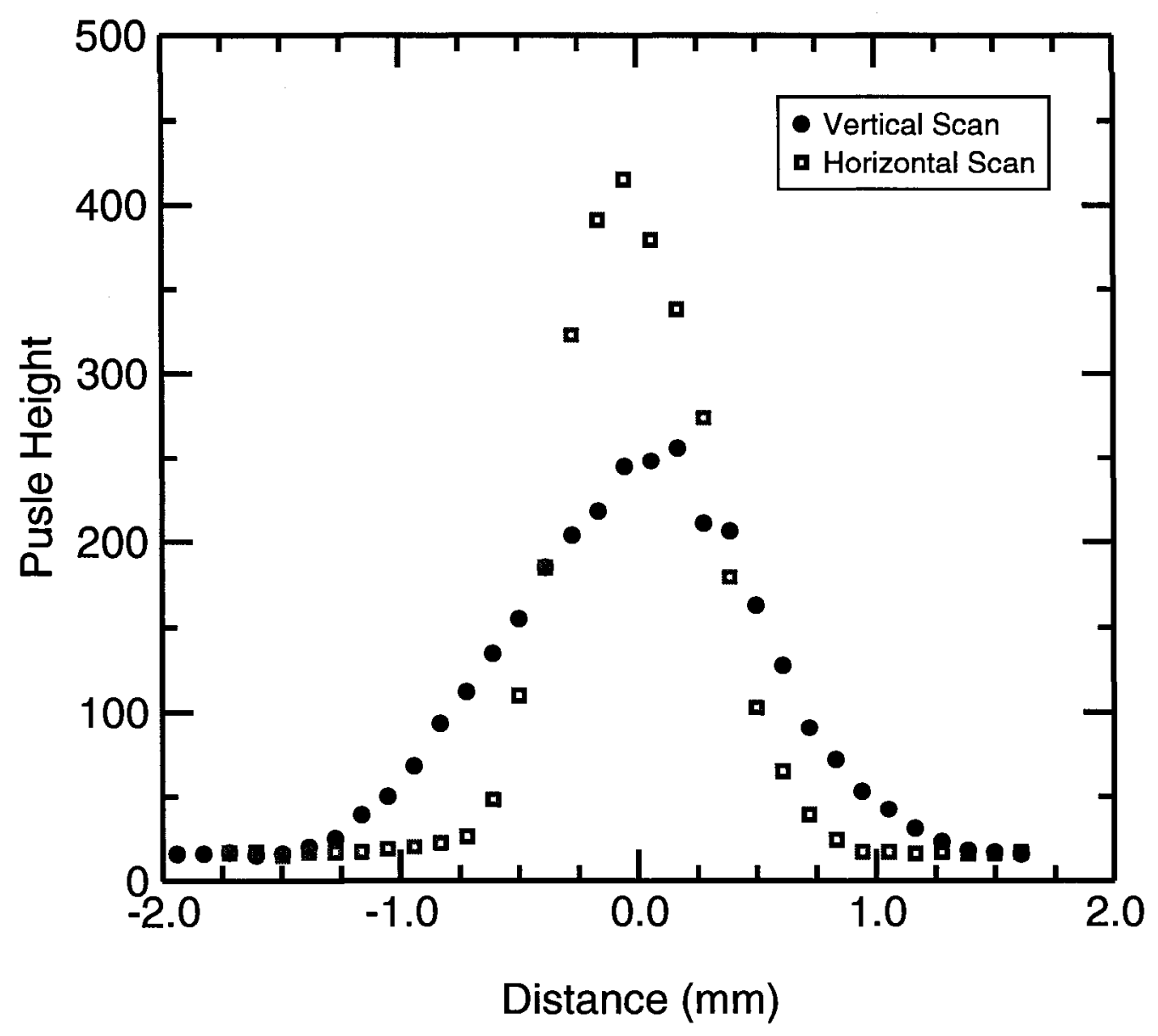

Figure 5.3: Vertical and horizontal profile scan of the $20 \mathrm{MeV}$ input beam. 
resulted in a small increase of $0.3 \%$ in the characteristic angle as seen in Table 5.1. This confirms a previous study which showed that the initial beam diameter does not affect the mean angular scatter (Blais \& Podgorsak, 1992). Therefore typical variations in the initial beam diameter do not affect the characteristic angle as shown.

The input energy for the Vickers linac is tunable using the beam transport bending magnets. During the experimental trial, the beam magnets were left untouched and over the course of a typical measurement duration the beam energy was constant. The beam energy was calibrated with an uncertainty of $\pm 0.4 \%$ at the exit window (MacPherson \& Ross, 1998). Therefore the effect of the beam energy on the characteristic angle had to be addressed. Firstly, the beam energy was tuned from $20 \mathrm{MeV}$ to $13 \mathrm{MeV}$. This showed an increase of the characteristic angle by $\approx 50 \%$ for the 'window only' case or a variation of $7 \%$ per MeV. Thus, if the beam energy varies by $0.4 \%$, (a variation of $0.08 \mathrm{MeV}$ at $20 \mathrm{MeV}$ ), this will result in a $0.5 \%$ variation in the characteristic angle.

A Monte Carlo investigation of changing the initial beam energy on a gold scattering foil $\left(218.7 \mathrm{mg} / \mathrm{cm}^{2}\right)$ resulted in a variation of the characteristic angle. The characteristic angle decreased by $5 \%$ as the energy of the initial beam was increased by $5 \%$. This can be seen in Figure 5.4. The characteristic angle changed by $0.4 \%$ for a variation in the beam energy of $0.4 \%$ or $0.08 \mathrm{MeV}$ at $20 \mathrm{MeV}$. This was similar to the experimental results.

The last beam characteristic to be investigated was the mean angular spread of the initial beam. As discussed previously, the mean angular spread has a significant impact on the characteristic angle. As the mean angular spread increases, the characteristic angle also increases. This can be seen from Equation 2.7. Unfortunately, this experimental measurement is more difficult than the other beam characteristics as it involves modification of the linac. Therefore an upper limit was estimated and a Monte Carlo investigation 
Table 5.1: Investigation of the variation of the characteristic angle with initial beam diameter (FWHM).

\begin{tabular}{|c|c|}
\hline $\begin{array}{c}\text { Initial beam diameter } \\
\text { (FWHM in } \mathrm{mm})\end{array}$ & $\theta_{c}\left(^{\circ}\right)$ \\
\hline 1.0 & 1.440 \\
2.0 & 1.442 \\
3.0 & 1.442 \\
4.0 & 1.446 \\
\hline
\end{tabular}

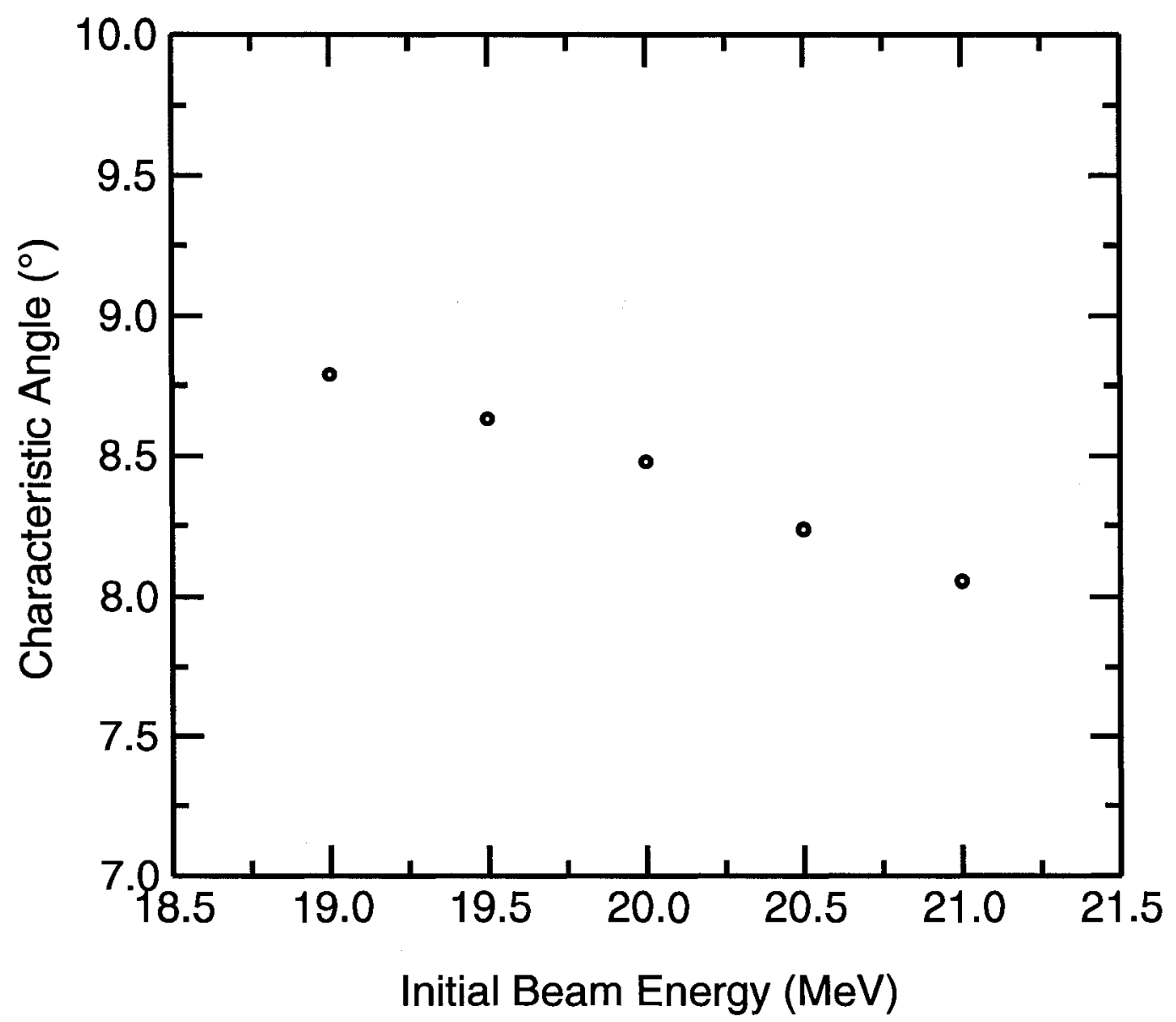

Figure 5.4: Relationship of the characteristic angle for a gold foil $\left(218.7 \mathrm{mg} / \mathrm{cm}^{2}\right)$ and the initial beam energy. 


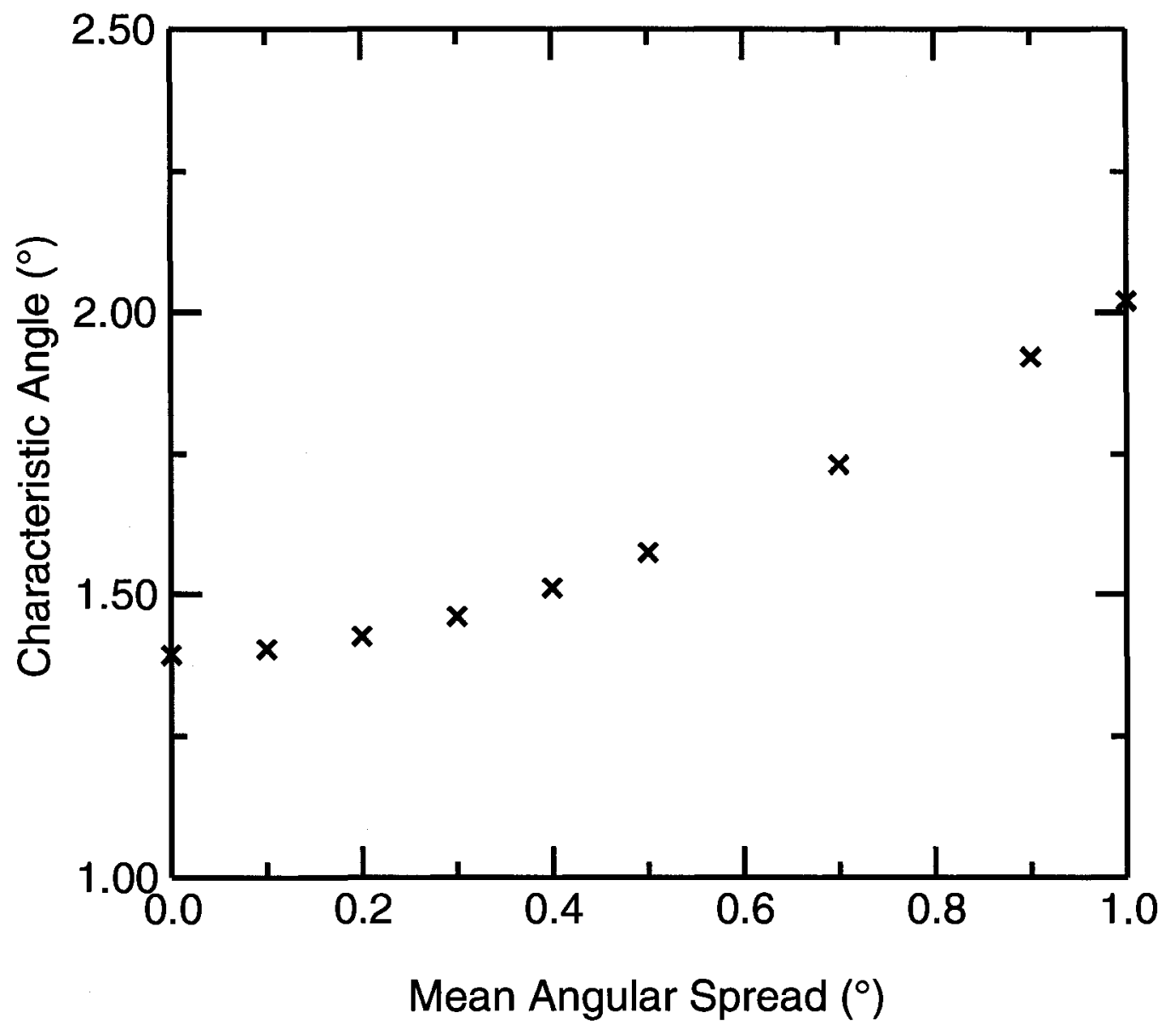

Figure 5.5: Relationship between the characteristic angle for a titanium alloy (18.21 $\mathrm{mg} / \mathrm{cm}^{2}$ ) and mean angular spread. 
was performed. For the Monte Carlo investigation, the initial mean angular spread was increased. The direct consequence of this variation can be observed in the characteristic angle. When an increase of the mean angular spread by $1^{\circ}$ occurs, the characteristic angle increases by almost $50 \%$ for a single titanium alloy foil $\left(18.21 \mathrm{mg} / \mathrm{cm}^{3}\right)$ as seen in Figure 5.5.

The following experimental measurement could be carried out to determine the mean angular spread of the beam. Radiochromic film could be used to measure the size of the beam at the exit window. Then an evacuated $1.0 \mathrm{~m}$ beam drift pipe could be added at the exit window location and the same measurement of the beam with radiochromic film would be performed. One should be able to measure the size of the mean angular spread using the difference in the size of these beam diameters on the films.

\subsection{Spatial Resolution}

There are many different types and models of detectors which are commercially available. For this study, ion chambers and diodes were investigated. The ion chambers used span volumes from a $0.009 \mathrm{~cm}^{3}$ micro-ion chamber to a $0.25 \mathrm{~cm}^{3}$ ion chamber. The diodes were chosen from two manufacturers because they were available at NRC at the time. An investigation of the detectors showed that the measured characteristic angle was dependent on the diameter of the sensitive region. This can be seen as the circular points in Figure 5.6. As the diameter of the sensitive region increases, the measured characteristic angle also increases. This can be explained as the convolution of two profiles: the result of the electron scattering and the averaging over the detector diameter. With a larger detector diameter, the characteristic angle of the final result will be inherently larger.

The investigation also showed that all of the diodes were dose-rate dependent. The SFD (stereotactic field detector) showed a less than $1 \%$ dose-rate dependence, but the 


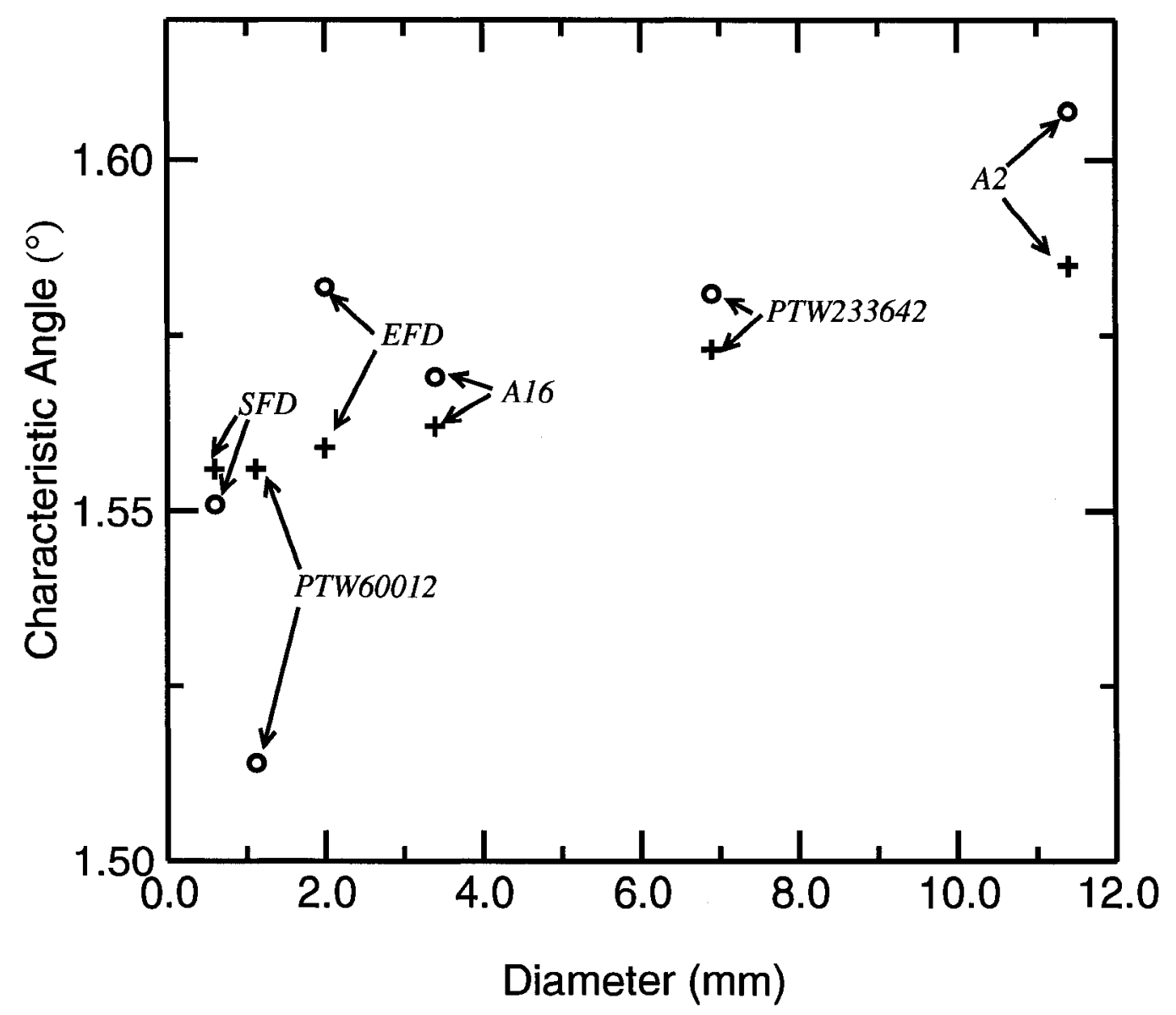

Figure 5.6: Results of an investigation of various detectors and effects of the measured characteristic angle. The circles are the uncorrected data and the crosses are the corrected data. These were corrected for both dose-rate dependence (diodes) and ion recombination (ion chambers). 
measured electron scattering profiles were noisy and made for diffcult extraction of the characteristic angle. However, the dose-rate dependence of the EFD (electron field diode) detector was small and with additional measurements the dose-rate dependence was characterized. The characteristic angle was corrected for this dose-rate dependence when required. The corrected characteristic angle can be seen in Figure 5.6 for the diodes and ion chambers investigated (crosses). The correction for the diodes was the dose-rate dependence and for the ion chambers was the ion recombination which will be discussed later in Section 5.5 on charge collection influence quantities. This dose-rate dependence was not documented in the specification sheets supplied by the manufacturer or available on the company website for any of the diodes.

Spatial resolution was also an issue with the Monte Carlo simulations. When scoring the dose, one has to choose an appropriate bin size best suited for the situation. The bin size should not significantly affect the characteristic angle and based on Figure 5.6, a bin size of $5 \mathrm{~mm}$ was used.

\subsection{Corrections for Influence Quantities}

All of the experimental measurements were carried out using the Exradin A16 micro-ion chamber and the Scanditronix EFD diode as the scanning detectors. The Elekta monitor chamber was used as the transmission monitor chamber for all trials.

Over a typical measurement run, the charge collected in the transmission monitor chamber varied up to $5 \%$ as previously seen in Figure 3.6. This variation in the output beam affects the amount of charge collected in the detectors. However, since the response of the scanning detector was taken relative to the transmission monitor chamber response, this variation in the beam will not affect the characteristic angle. 


\subsubsection{Charge collection contributions}

The corrections addressed in this section will focus on the charge collection within the detectors. These corrections are predominately for the A16 and Elekta monitor ion chambers since these are vented detectors. Some of these corrections are not applicable to solid state diode detectors. During a measurement, the raw charge is collected in the detectors. However, the dose to the medium required a corrected charge collection. There are a few corrections which can be applied to this measurement including the effects from the temperature and pressure, ion recombination and polarity.

There was no temperature correction needed for the A16 micro-ion chamber measurements. Since the measurement was a relative response to the Elekta monitor chamber, any temperature variation in the experimental hall would require the same correction for both of the ion chambers. The EFD diode is not a vented chamber, therefore the measurements with the diode would usually not require an air temperature correction.

The second part of the air density correction is the pressure correction. The same situation as the temperature correction for the ion chamber applies. Since both chambers collect charge and the profile is based on the relative response, the pressure correction cancels out. The pressure of the environment affects the collected charge in the monitor chamber but not the solid state diode. However, a pressure correction was simply a scaling factor to the charge collected in the monitor chamber, so again a correction was not required. The only way a correction would be required is if the pressure or temperature (or both) varied dramatically over the course of the 10-15 minutes measurement run. An investigation of this was performed and a linear increase of the correction $P_{T P}$ of $5 \%$ (impossibly large) over the measurement run had an effect of $0.01 \%$ on the characteristic angle. With the data acquisition procedure of interleaving the experimental data points, the result is insensitive to a large environmental variation. 
Two sources of measurement background come from natural radiation and detector leakage. The background radiation effects on the detectors are very small, and were are not measurable. The main source of background was the ion chamber insulator leakage. In the set-up, sources for the leakage were the $40 \mathrm{~m}$ cable from the electrometer in the control room to the detector in the experimental hall and the electrometer/ion chamber combination. Measurements showed that there was no significant detector leakage, therefore no correction was required.

An investigation of the ion recombination showed that the A16 micro-ion chamber has a small correction, under $1 \%$. This correction affects the characteristic angle. The ion recombination is proportional to the dose per pulse and over the profile the correction will vary based on the charge collected. Therefore at the peak a larger correction is required and the corrected profile will become narrower and decrease the characteristic angle. However, the small required correction only affected the two narrowest profiles which were the thin titanium alloy scattering foils.

The polarity effect for the A16 micro-ion chamber was measured using a similar technique to that described in Section 2.5. A profile was obtained while collecting positive charge and then again while collecting negative charge. These were used to investigate the polarity correction over the profile as shown in Figure 5.7. This seems to be a slight trend from left to right, but with the procedure of interleaving the data points a direct cause of this trend is not apparent. However, the extraction of the characteristic angle is from $-4 \mathrm{~cm}$ to $+4 \mathrm{~cm}\left(\mathrm{or} \approx 2^{\circ}\right.$ ) which shows a very small variation in the polarity effect. The correction was found to have a variation less than $0.2 \%$ over the range of the scanned profile and, therefore, no correction was applied.

The measurement repeatability from day-to-day for the same set-up had a variation of $0.3 \%$ in the characteristic angle. 


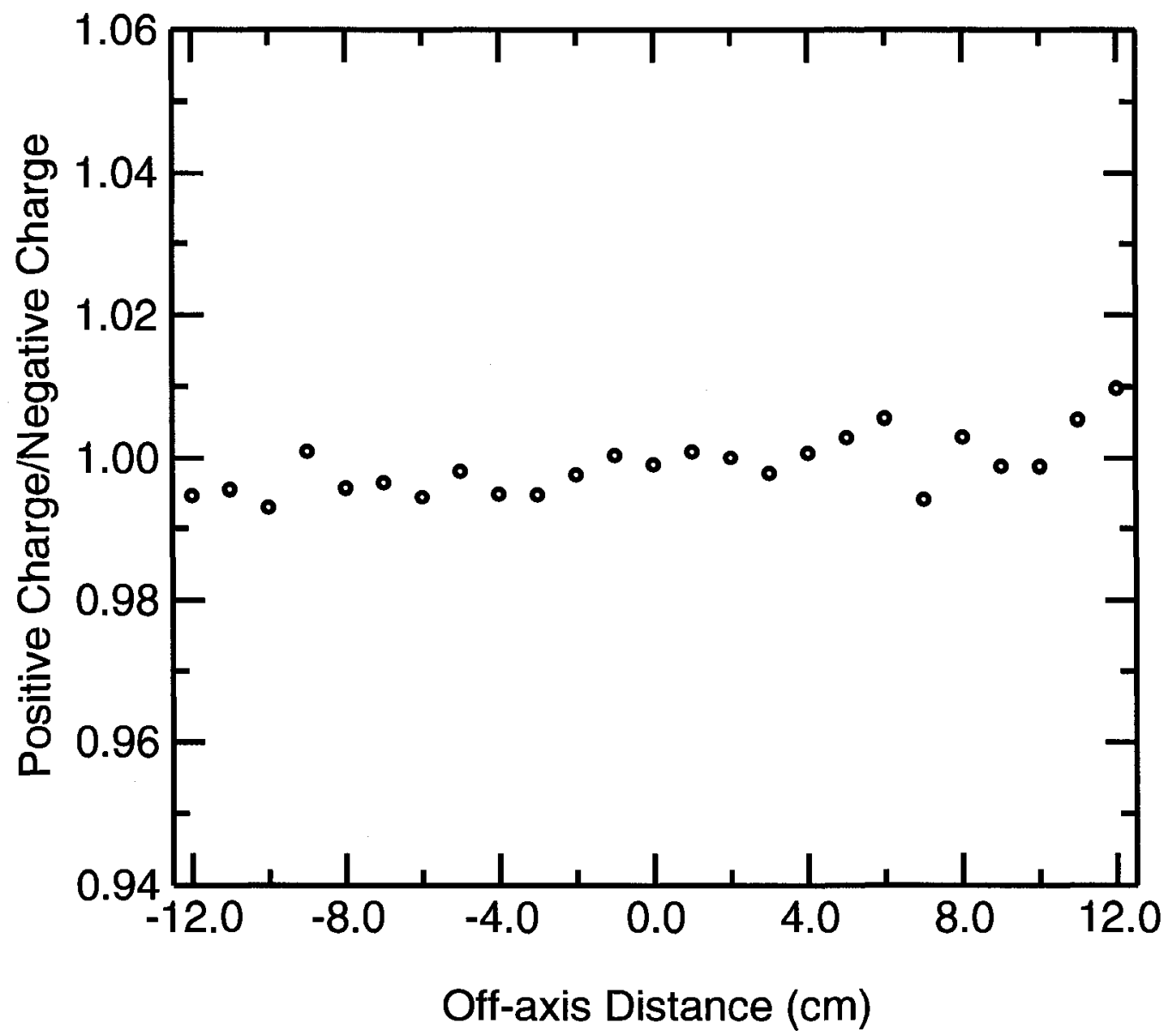

Figure 5.7: The ratio of positive charge collected to negative charge collected over the scanning range for the simple scattering case of the A16 micro-ion chamber. 


\subsubsection{Secondary radiation}

For measurements of a high energy electron beam, there can be secondary radiation collected in the detectors from knock-on electrons or bremsstrahlung. The goal of this study was to measure the electron scattering profiles of a primary electron beam. Therefore the effects of secondary radiation on the measurements were investigated.

An experimental investigation of these secondary effects was performed using the main detectors (EFD and A16) with a lead build-up cap. The lead build-up cap was made thick enough to stop the primary electron beam and enabled the bremsstrahlung to be measured in the detector. This measurement was carried out for a select number of scattering foils. Using a $10 \mathrm{~mm}$ lead cap, the bremsstrahlung contamination was measured to be $3 \%$ of the peak of the profile. Unfortunately, while these measurements were possible, they were inconclusive as they only showed an upper limit on the bremsstrahlung. A significant proportion of the bremsstrahlung was produced in the lead cap. A second investigation of the bremsstrahlung was performed using an electromagnet. This electromagnet replaced the drift region Mylar bag and was used to deflect the electrons away from the scanning detector. Thus, only the bremsstrahlung would be measured. Again, this technique did not allow for a definitive answer to the bremsstrahlung measurement with a $0.8 \%$ upper limit, as the electron scattering was too large for all the electrons to be swept away from the detector.

A more thorough investigation of secondary radiation was performed using Monte Carlo simulations. Using DOSRZnrc, the particle types at the measurement plane were investigated. Using this feature, the dose from the electrons and the photons was scored and compared. This can be seen in Figure 5.8. The photon dose is less than $0.1 \%$ of the electron dose over the measurement range.

Another Monte Carlo simulation to investigate the secondary radiation was to calcu- 


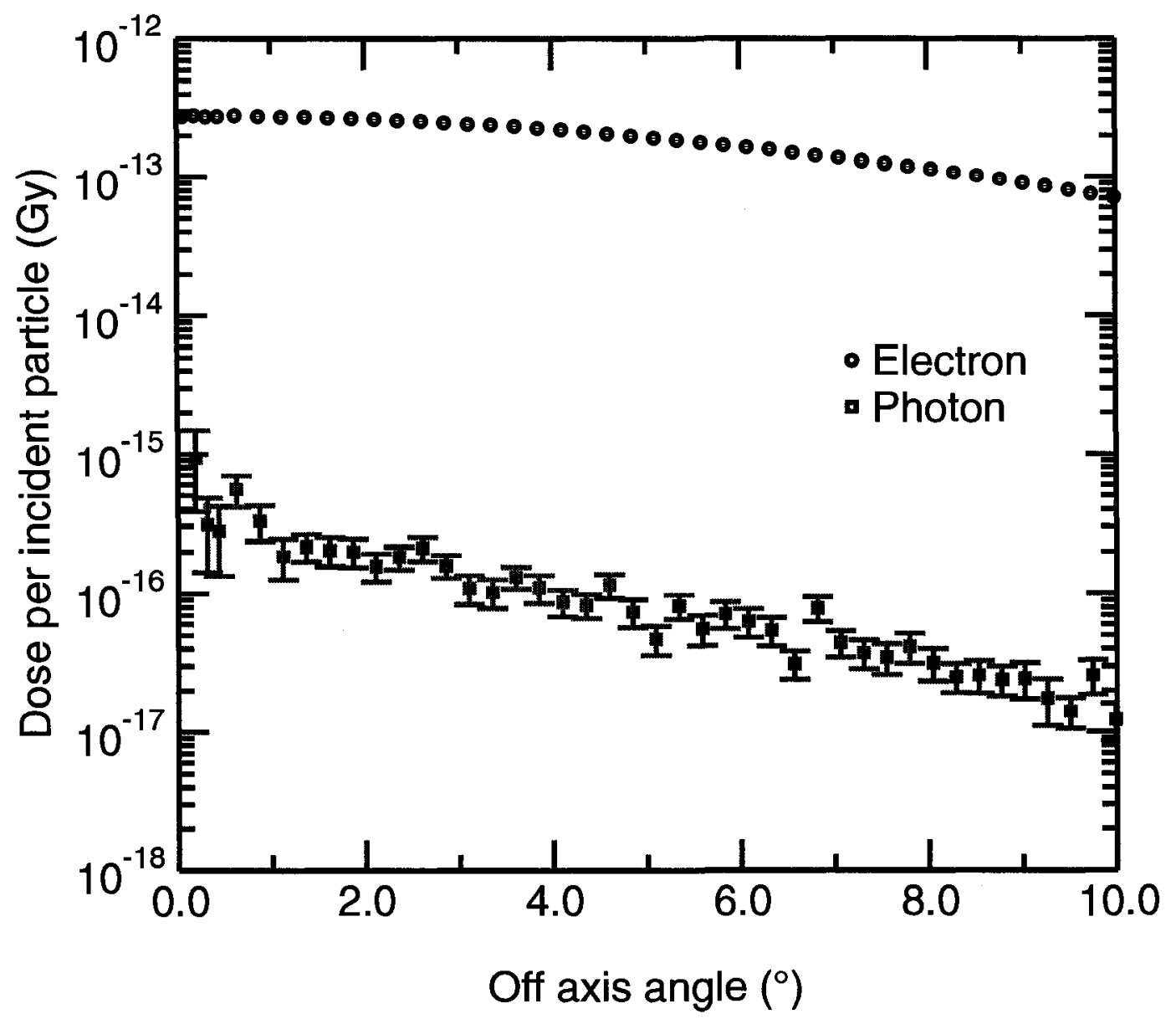

Figure 5.8: A Monte Carlo investigation of the dose scored from electrons and photons produced by a gold foil $\left(218.7 \mathrm{mg} / \mathrm{cm}^{2}\right)$ 


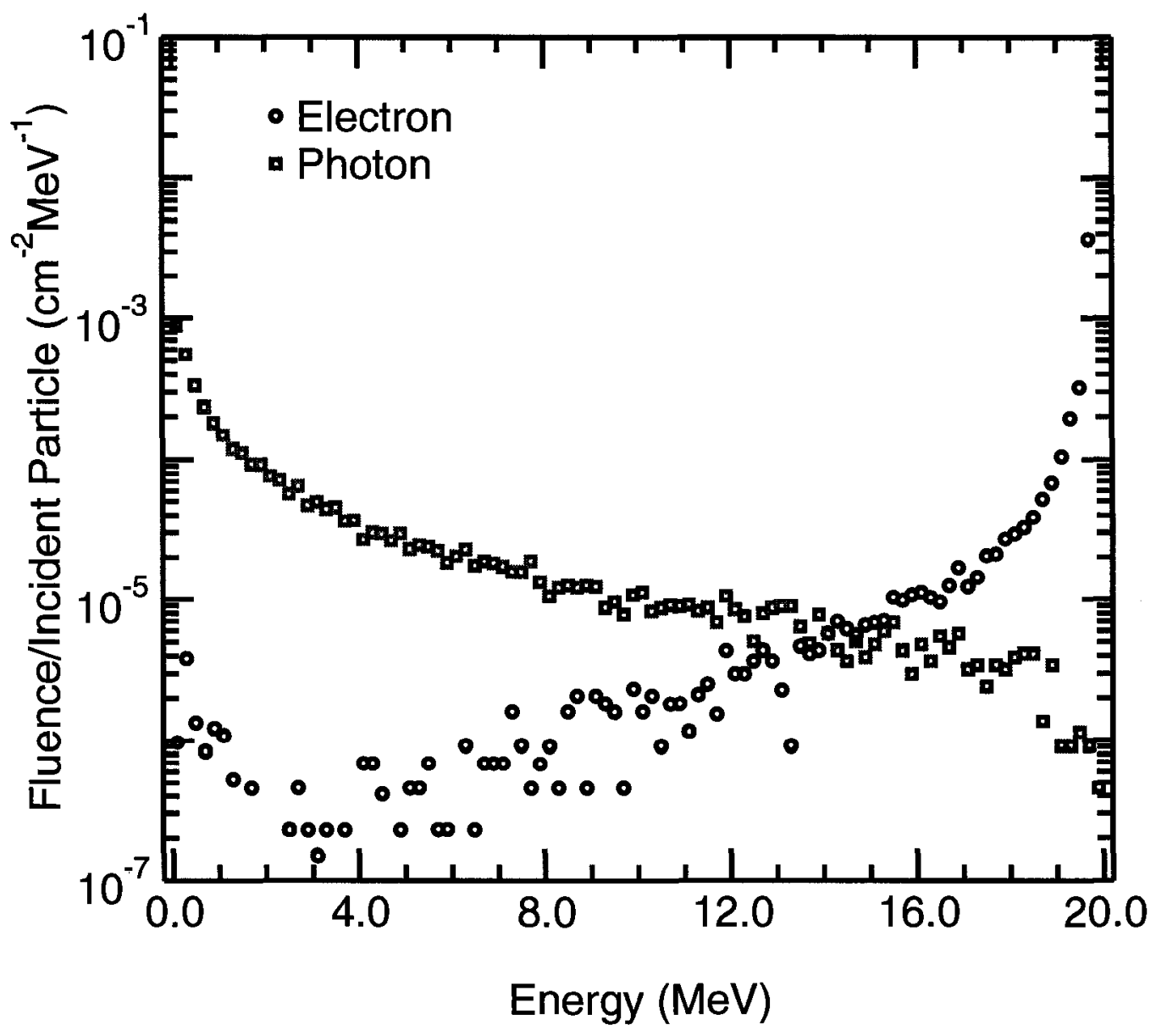

Figure 5.9: A Monte Carlo investigation of the energy fluence at the measurement plane for electrons and photons produced by a gold foil $\left(218.7 \mathrm{mg} / \mathrm{cm}^{2}\right)$. Scoring bin was on beam axis. 
late the energy fluence of the electrons and photons. The simulation showed the energy of the particles at the measurement plane for a gold scattering foil seen in Figure 5.9. The majority of the electrons have lost very little energy with a quick drop off of the number of electrons at lower energies. At high energy, the photon fluence looks similar to a typical bremsstrahlung yield with photons at the same energy as the incident particles and increase in numbers to a peak. However, the number of photons continues to increase at lower energies. This increase can be attributed to the scattered photons. The difference in the measured dose in Figure 5.8 from the photons and the number of photons at the measurement plane as seen in Figure 5.9 is because the probability of interaction for photons is significantly lower than the probability of interaction for electrons.

\subsection{Uncertainties}

All of the known sources of uncertainties have been addressed in the previous sections. As discussed, there are many sources of potential uncertainties: the beam characteristics, the detector characteristics, measurements and the analysis of the data. Each of the uncertainties can also contribute a different amount depending on the width of the profile, seen in Table 5.2.

From Equation 2.3 and Equation 2.4, it can be seen that the mean square angle of scattering (or the square of the characteristic angle) of the profile is proportional to $1 / E^{2}$, where $E$ is the energy of the initial beam. As discussed in Section 5.3 the initial beam energy uncertainty is $0.4 \%$ and this would mean that the uncertainty in the characteristic angle would also be $0.4 \%$. An experimental and Monte Carlo investigation were performed and also showed that the uncertainty in the characteristic angle would be $0.5 \%$ and $0.4 \%$, respectively.

The next beam characteristic to consider is the beam diameter and a Monte Carlo in- 
vestigation showed that an increase of the initial beam FWHM from $1.0 \mathrm{~mm}$ to $2.0 \mathrm{~mm}$ for the $20 \mathrm{MeV}$ simple scattering case increased the characteristic angle $0.07 \%$. This increase in the characteristic angle is not a significant increase and, therefore no uncertainty is assigned.

The uncertainties associated with the mean angular spread of the beam are harder to assign due to the lack of experimental measurements of the mean angular spread. However, given the Monte Carlo investigation of the mean angular spread and the experimental upper-limit from the beam transport, an estimated uncertainty can be assigned. The experimental upper-limit of the mean angular spread was $0.72^{\circ}$. This is a maximum value and a reasonable estimate of the mean angular spread based on the ISO guide to measurement uncertainties, would be $0.21^{\circ}$ (ISO, 1995). This is based on the fact that the value of the mean angular spread lies between $0.72^{\circ}$ and $0^{\circ}$ with probability of 1 and lies outside this range with a probability of 0 . If this estimate of the mean angular spread is correct, then all the Monte Carlo calculated characteristic angles would have an additional $\left(0.21^{\circ}\right)^{2}$ (from Equation 2.7). A large uncertainty in the mean angular spread will dominate the overall uncertainties. Therefore, this was not added to the uncertainty budget but will be included in the discussion of the results.

When measuring the mass thickness of the foils, there are also uncertainties which can have an impact on the results. The thickness of the foil is difficult to measure directly, therefore the area and the mass were used to compute the mass thickness. Not only does the mass thickness affect the experimental characteristic angle, it has an impact on the Monte Carlo calculations.

The BEAMnrc input file does not allow for the input of the experimental mass thicknesses directly. The user defines linear thicknesses for each material and the mass thickness is calculated from the PEGS data set material densities. The linear thickness was 
therefore adjusted to give the same mass thickness as determined experimentally. For the simulations, the scattering foil is the main contribution to the overall scattering therefore the simulations needs to have the best estimate of the scattering foil mass thickness. If the experimental measurement and Monte Carlo calculation have different values of the mass thickness this will lead to a difference in the characteristic angle. It can be seen in Figure 5.10 that for a $5 \%$ increase in foil thickness the characteristic angle increased by $2.1 \%$.

The detector resolution uncertainty is associated with the finite size of the detectors and will only affect the narrow profiles. This uncertainty will only affect the absolute characteristic angle and drop out when comparing the Monte Carlo calculations because the finite size of the dose bins are about the same size as the detector sizes.

The detector response uncertainties are attributed to the charge collection, mainly the ion recombination of the ion chamber and the dose-rate dependence of the diode. Again, with a broader profile the correction will be smaller, therefore the uncertainty will also be smaller.

Positioning of the detector at the correct distance from the exit window will affect the angle directly. The off axis angle is based on the distance from the exit window of $118.2 \mathrm{~cm}$. However, there is some uncertainty with this repositioning for each of the detectors.

The fitting process also will affect the characteristic angle, as seen in Section 5.2. The uncertainty of the fitting procedure was estimated by a fit of the profile from an automated fitting package (SigmaPlot 10.0) and comparing this to the technique used in this study. This uncertainty is $0.5 \%$ based on the fitting technique.

Table 5.2 collects the estimated standard uncertainties $\left(\mathrm{u}_{i} \%\right)$ associated with the characterstic angle from various sources. 


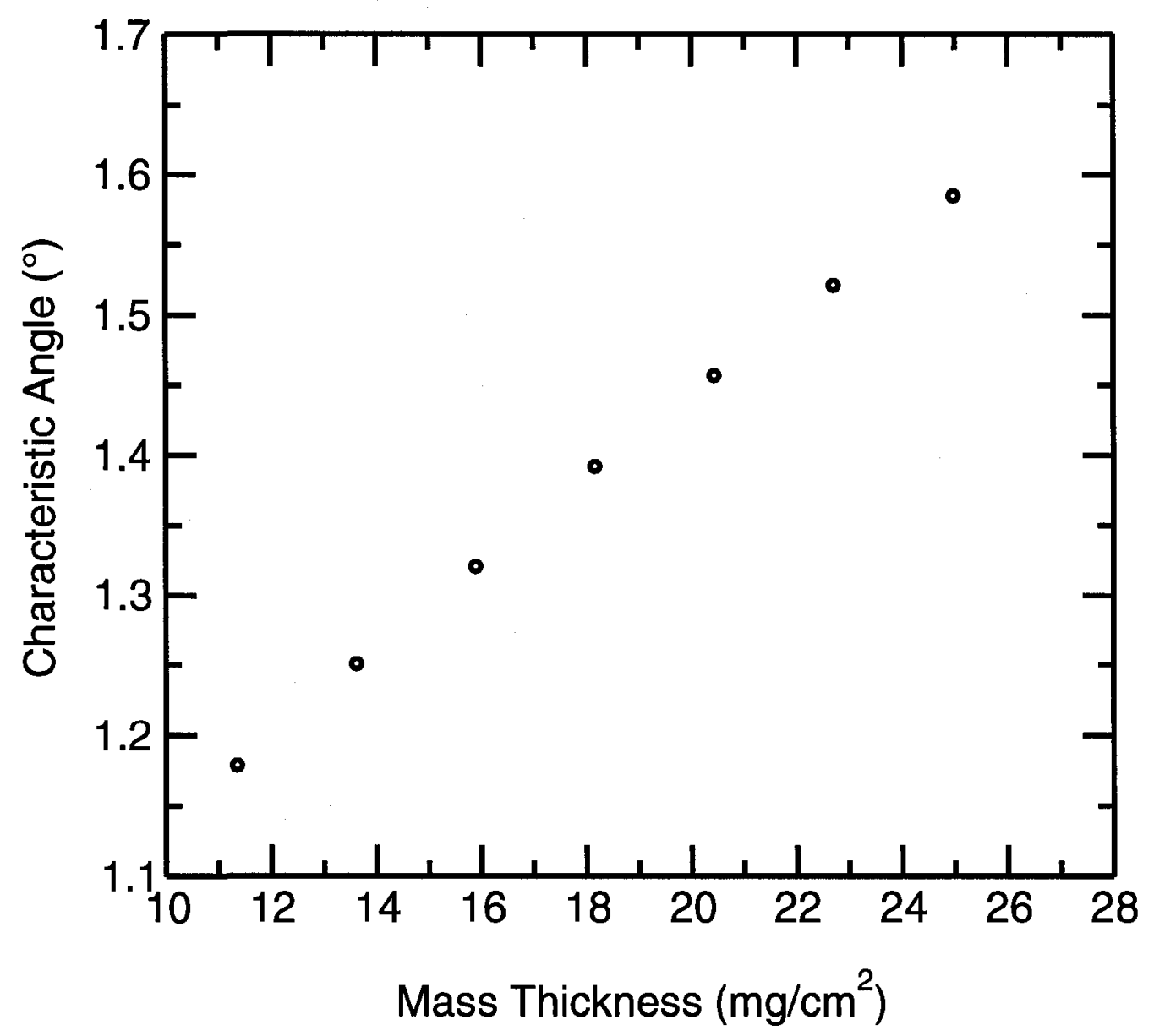

Figure 5.10: A Monte Carlo investigation of the mass thickness sensitivity for comparisons of the characteristic angle using a titanium alloy series. 
Table 5.2: Uncertainties associated with the characteristic angle

\begin{tabular}{|l|c|c|c|c|}
\hline \multirow{2}{*}{ Uncertainty } & $20 \mathrm{MeV}$ Window & $\theta_{c} \approx 2^{\circ}$ & $\theta_{c} \approx 4^{\circ}$ & $\theta_{c} \approx 8^{\circ}$ \\
\cline { 2 - 5 } & $\mathrm{u}_{i}(\%)$ & $\mathrm{u}_{i}(\%)$ & $\mathrm{u}_{i}(\%)$ & $\mathrm{u}_{i}(\%)$ \\
\hline Beam energy & 0.4 & 0.4 & 0.4 & 0.4 \\
Beam alignment & 0.2 & 0.1 & 0 & 0 \\
Foil thickness & 0.25 & 0.25 & 0.25 & 0.25 \\
Detector resolution & 0.3 & 0.17 & 0.04 & 0.0 \\
Detector positioning & 0.3 & 0.3 & 0.3 & 0.3 \\
Detector response & 0.5 & 0.3 & 0.2 & 0.2 \\
Repeatability & 0.3 & 0.3 & 0.3 & 0.3 \\
Fitting & 0.5 & 0.5 & 0.5 & 0.5 \\
\hline Quadratic sum & 1.02 & 0.88 & 0.83 & 0.83 \\
\hline
\end{tabular}

\subsection{Results}

The mass thickness, characteristic angle of the profile, the fitted data range for experimental measurements and Monte Carlo calculations of electron scattering profiles for 13 and $20 \mathrm{MeV}$ electron beams are shown in Table 5.3 and Table 5.4, respectively. Measurements were taken for a series of elements, titanium alloy, copper and gold, for several mass thicknesses of each. Monte Carlo calculations were completed for each series and at both energies. When comparing results between various set-ups, for example, experimental measurements and Monte Carlo calculations the quantity of comparison needs to be the same. In this case, the measurement and calculation of dose were compared. 

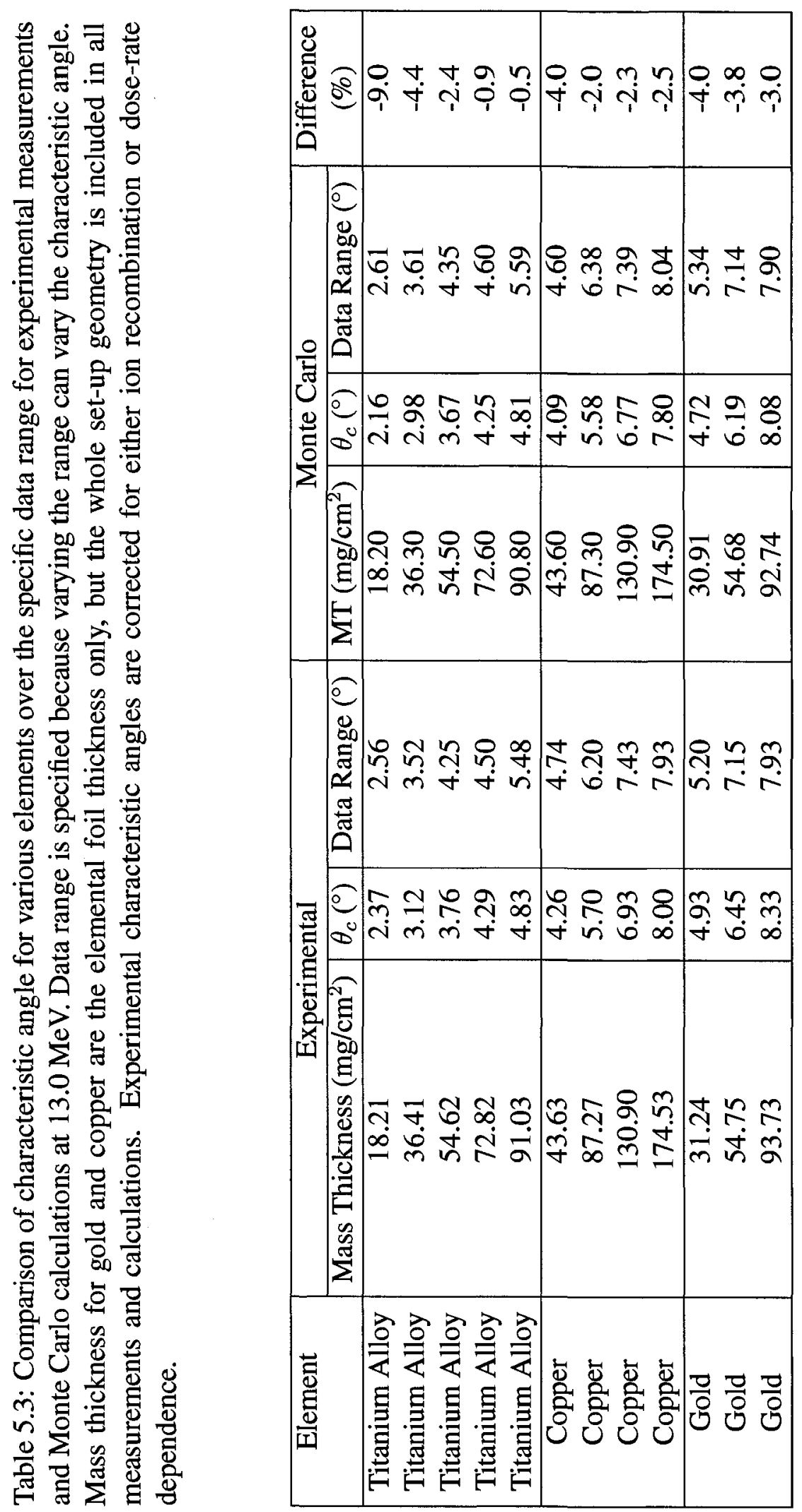

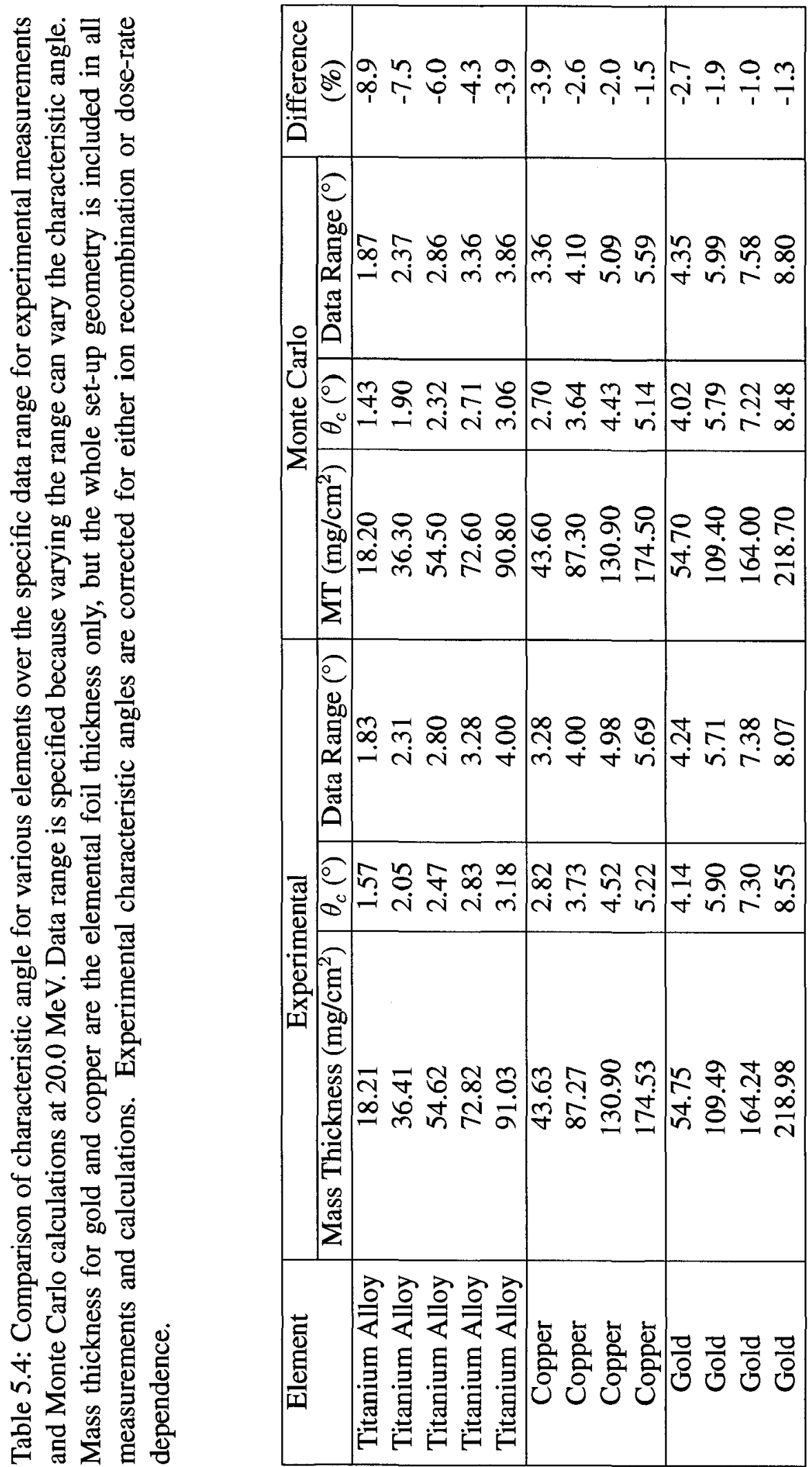


\subsection{Discussion}

The difference between the measured and calculated characteristic angle is also contained within the tables. This difference was calculated by way of $\left(\theta_{c}^{\mathrm{MC}}-\theta_{c}^{\mathrm{EXP}}\right) / \theta_{c}^{\mathrm{EXP}}(\%)$. The experimental data set has been characterized and uncertainties of the set-up and measurements have been addressed. The measurements had uncertainties around $1 \%$, which was the aim of the study. The associated uncertainties have been presented in Table 5.2.

There is a trend of decreasing difference with increased mass thickness. However, this trend is not present with atomic number. All of the differences are negative which needs to be addressed. This could be from an additional small scatter in the experimental set-up, which was not modeled in the Monte Carlo simulation. A scatter will have the biggest effect on the narrower profiles. The measurements of the mean angular spread will provide a source to broaden the profile and will reduce this difference. The required angle to reduce the difference to zero for each foil is given in Table 5.5. This angle would give insight to how much 'extra scattering' would be required for each case. If the angle was about the same this would show that there was a general lacking of a scattering element in the simulation. For all cases the required angle is larger than the maximum possible mean angular spread of the beam. There is also a trend of increased required angle with mass thickness, and thus the mean angular spread of the beam is not the only solution to this difference.

An independent check of a subset of the Monte Carlo calculations (the copper series at $20 \mathrm{MeV}$ ) was performed by Claudiu Cojocaru at NRC and both the Monte Carlo calculations results agree to $0.03 \%$.

The main comparison between the Monte Carlo calculations and the experimental measurements are with the characteristic angle obtained from the Gaussian fit. However, this does not investigate the differences over the whole profile. The shape of the profile is 
Table 5.5: Angle required to elimination the difference between the Monte Carlo calculations and experimental measurement of the characteristic angle.

\begin{tabular}{|c|c|c|c|}
\hline \multirow{2}{*}{ Element } & Mass Thickness $\left(\mathrm{mg} / \mathrm{cm}^{2}\right)$ & \multicolumn{2}{|c|}{ Angle Required $\left(^{\circ}\right)$} \\
\cline { 3 - 4 } & & $13 \mathrm{MeV}$ & $20 \mathrm{MeV}$ \\
\hline Titanium Alloy & 18.21 & 0.98 & 0.65 \\
Titanium Alloy & 36.41 & 0.92 & 0.77 \\
Titanium Alloy & 54.62 & 0.82 & 0.85 \\
Titanium Alloy & 72.82 & 0.58 & 0.82 \\
Titanium Alloy & 91.03 & 0.44 & 0.87 \\
\hline Copper & 43.63 & 1.19 & 0.81 \\
Copper & 87.27 & 1.16 & 0.81 \\
Copper & 130.90 & 1.48 & 0.90 \\
Copper & 174.53 & 1.78 & 0.91 \\
\hline Gold & 31.25 & 1.42 & - \\
Gold & 54.75 & 1.81 & 0.99 \\
Gold & 93.73 & 2.03 & - \\
Gold & 109.49 & - & 1.13 \\
Gold & 164.24 & - & 1.08 \\
Gold & 218.98 & - & 1.09 \\
\hline
\end{tabular}


also a good comparison of overall Monte Carlo calculations and experimental measurements. If the profiles follow the same shape as the Monte Carlo calculational models of the experiment, then a scaling factor can be used to show the same shape. A plot of natural logarithm of the normalized relative response versus the square of the off-axis angle for all the collected data points can be seen in Figure 5.11.

A scaling factor of 1.1, found via visual inspection, was applied to the square of the off-axis angle of the Monte Carlo calculations. It can be seen that this scaled Monte Carlo profile is in good agreement with the experimental measurements. This scaling factor is about a $3 \%$ difference over the whole of the profile which is consistent with the difference in the characteristic angle.

Another comparison of Monte Carlo codes, including EGSnrc, to experimental measurements of thin foil electron scattering [Kulchitsky \& Latyshev (1942) and Hanson et al. (1951)] was performed by Vilches et al. (2007). Vilches et al. showed that EGSnrc underestimated the Kulchitsky \& Latyshev data set over the range of atomic number from $-6.5 \%$ to $-8.7 \%$. While the experimental measurements were made at $2.25 \mathrm{MeV}$, lower then the clinical energy range, Vilches et al. results are similar to those found in this study; differences are large and negative at lower energies. EGSnrc was in good agreement with both the Hanson et al. gold and beryllium foil measurements.

At this stage it is not possible to identify the reason for the difference between the measurement and the Monte Carlo results and further work is required. Such work on the electron scattering profiles could include measurements of the mean angular spread as proposed, improved measurement of the electron beam energy, and improved foil measurement. With additional information gathered on the incident beam, a more correct Monte Carlo calculation can take place. Also the correct construction of the Elekta monitor chamber should be verified and implemented in the simulation. The comparison 


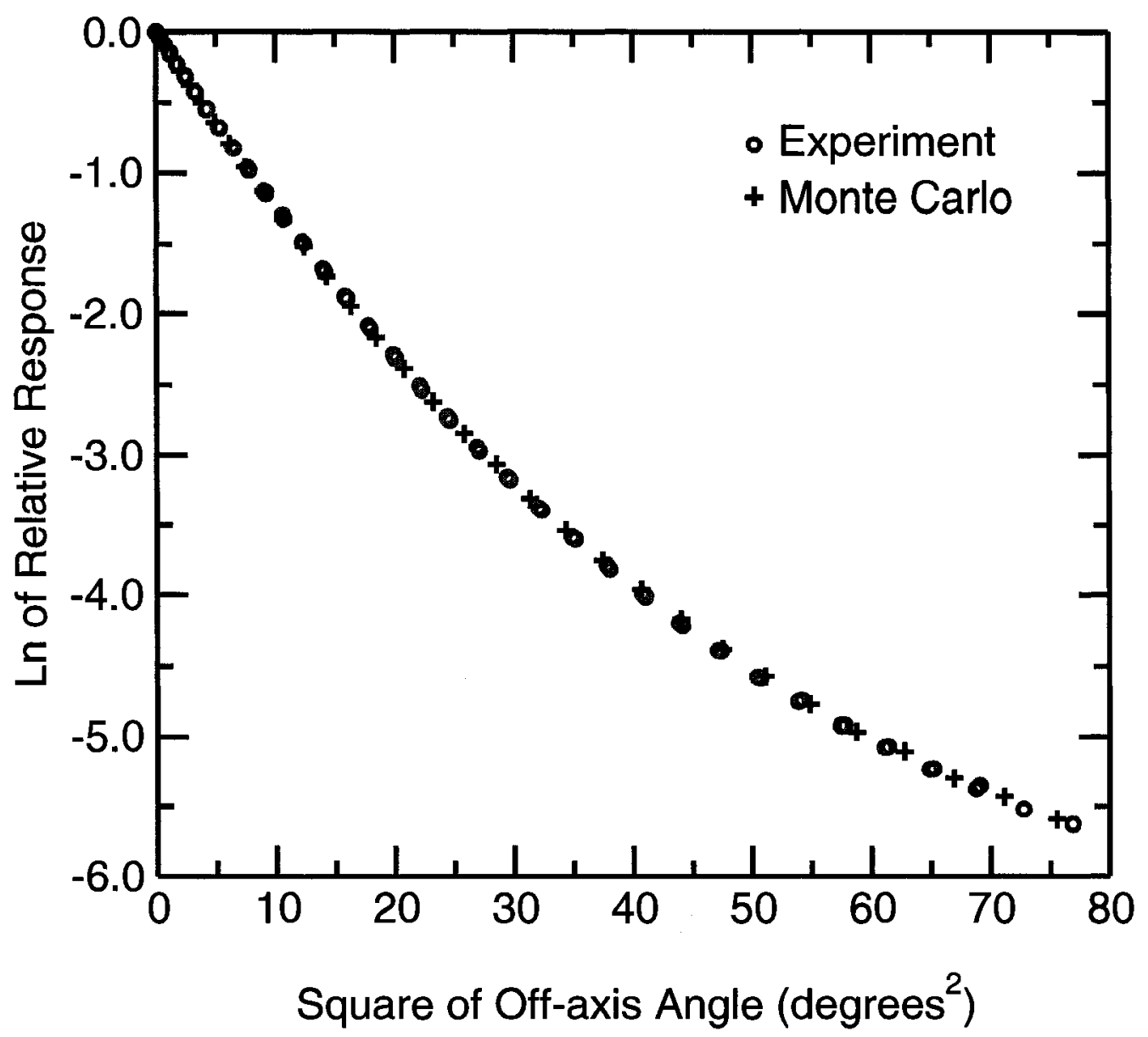

Figure 5.11: Comparison of the natural logarithm of the normalized relative response versus the square of the off-axis angle for experimental measurements and scaled Monte Carlo calculations of a thin copper foil $\left(43.63 \mathrm{mg} / \mathrm{cm}^{2}\right)$ at $20 \mathrm{MeV}$. The off-axis angle squared of the Monte Carlo calculations have been scaled by 1.1. 
routine of the profiles also can be improved by using the full experimental data set of each scan. A routine which would compare the whole of the profile should give improved understanding of the reasons for the difference between Monte Carlo calculations and the experimental measurements. To further investigate the accuracy of the Monte Carlo model, it could be used to simulate the Kulchitsky \& Latyshev and Hanson et al. data. 


\section{CHAPTER 6}

\section{Conclusions}

This study set out to measure electron scattering profiles for various elemental scattering foils at different mass thicknesses ranging from 20 to $220 \mathrm{mg} / \mathrm{cm}^{2}$ and incident beam energies of 13 and $20 \mathrm{MeV}$. The experimental profiles were described using the characteristic angle $\left(\theta_{c}\right)$, the angle at which the intensity is $1 / e$ the peak intensity or the characteristic angle of a Gaussian. Profile characteristic angles were measured from $1.57^{\circ}$ to $8.55^{\circ}$. Investigations of various initial beam and set-up parameters were performed.

The initial beam characteristics of the electron beam were studied prior to scattering foil measurements. The three main characteristics are: the beam energy, the beam diameter and the mean angular spread. The beam energy has been previously studied by MacPherson \& Ross (1998). The beam diameter was measured using a helical wire profile monitor to be $0.70 \mathrm{~mm}$ (FWHM of $1.17 \mathrm{~mm}$ ) in the vertical direction and $0.50 \mathrm{~mm}$ (FWHM of $0.83 \mathrm{~mm}$ ) in the horizontal direction. The mean angular spread of the initial beam was not measured; however, an upper limit based on the geometry of the beam transport was estimated to be $0.72^{\circ}$ at the exit window.

A well known set-up geometry was used for the study to identify areas of refinement for the electron scattering profile measurements and focus on the scattering from the thin foils.

Monte Carlo calculations of the experimental set-up were carried out using BEAMnrc and DOSRZnrc. Various geometry, foil and scoring configurations were modeled. The Monte Carlo calculations were also characterized using a similar technique to extract the characteristic angle over the same range as the experimental results. Differences between 
the measured and calculated profiles were found, ranging from $-0.5 \%$ to $-9.0 \%$. This difference could be due to incomplete Monte Carlo modeling of the set-up, and the mean angular spread of the incident beam was also identified as a possible component.

However, despite this apparent discrepancy, the measured electron scattering profiles are an excellent set of highly accurate experimental data for further use in Monte Carlo validation. With an overall uncertainty of $1 \%$ or less for all of the experimental measurements the main goal of the study was obtained. 


\section{REFERENCES}

Agostinelli, S., Allison, J., Amako, K., Apostolakis, J., Araujo, H., Arce, P., Asai, M., Axen, D., Banerjee, S., and Barrand, G. (2003). GEANT4 - a simulation toolkit. Nucl. Inst. Meth. in Phys. Res. A, 506, 250 - 303.

Aljarrah, K., Sharp, G. C., Neicu, T., and Jiang, S. B. (2006). Determination of the initial beam parameters in Monte Carlo linac simulation. Med. Phys., 33, 850-858.

Almond, P. R., Biggs, P. J., Coursey, B. M., Hanson, W. F., Huq, M. S., Nath, R., and Rogers, D. W. O. (1999). AAPM's TG-51 Protocol for Clinical Reference Dosimetry of High-Energy Photon and Electron Beams. Med. Phys., 26, 1847 - 1870.

Berger, M., and Seltzer, S. (1973). ETRAN Monte Carlo code system for electron and photon transport through extended media. Technical Report CCC-107, Oak Ridge National Laboratory, Oak Ridge, Tennessee.

Blais, N., and Podgorsak, E. B. (1992). The mass angular scattering power method for determining the kinetic energies of clinical electron beams. Phys. Med. Biol., 37, 1931 $-1942$.

Canadian Cancer Society (2007). Canadian Cancer Statistics. Technical Report ISSN0835-2976, National Cancer Institute of Canada, Toronto, Canada.

CERN Program Library Long Write-up (1993). GEANT - Detector description and simulation tool. Report W5013, Conseil Europèen pour la Recherche Nuclèaire, Geneva.

Chetty, I. J., Curran, B., J, E. C., DeMarco, J. J., Ezzell, G., Faddegon, B. A., Kawrakow, I., Keall, P. J., Liu, H., Ma, C. M. C., Rogers, D. W. O., Seuntjens, J., Sheikh-Bagheri, 
D., and Siebers, J. (2007). Issues associated with clinical implementation of Monte Carlo-based treatment planning: Report of the AAPM Task Group No. 105. Med. Phys..

Fernow, R. (1986). Introduction to Experimental Particle Physics. New York: Cambridge University Press.

Hanson, A. O., Lanzl, L. H., Lyman, E. M., and Scott, M. B. (1951). Measurement of multiple scattering of 15.7-MeV electrons. Phys. Rev., 84, 634- 637.

Haug, E., and Nakel, W. (2003). The Elementary Process of Bremsstrahlung. Singapore: World Scientific.

ICRU (1972). Radiation Dosimetry: Electrons with Initial Energies Between 1 and $50 \mathrm{MeV}$. ICRU Report 21, International Commission of Radiation Units and Measurements, Washington D.C.

ICRU (1984). Radiation Dosimetry: Electron beams with energies between 1 and $50 \mathrm{MeV}$. ICRU Report 35, International Commission of Radiation Units and Measurements, Washington D.C.

ISO (1995). Guide to the Expression of Uncertainties in Measurement. Geneva, Switzerland: International Standardization Organization.

Kawrakow, I., and Rogers, D. W. O. (2000). The EGSnrc Code System: Monte Carlo simulation of electron and photon transport. Technical Report PIRS-701, National Research Council of Canada, Ottawa, Canada.

Kovalev, V. P., Kharin, V. P., Gordeev, V. V., and Isaev, V. I. (1972). Angular distributions of electrons with initial energies of $12-25 \mathrm{MeV}$ behind tungsten, cadmium, and copper barriers. Atomic Energy, 33, 1088 - 1090. 
Kulchitsky, L. A., and Latyshev, G. D. (1942). The Multiple Scattering of Fast Electrons. Physical Review, 61(5-6), 254-265.

Li, X. A., and Rogers, D. W. O. (1995). Electron mass scattering powers: Monte Carlo and analytical calculations. Med. Phys., 22, $531-541$.

MacPherson, M. S., and Ross, C. K. (1998). A Magnetic Spectrometer for Electron Energy Calibration. Technical Report PIRS-617, National Research Council of Canada, Ottawa, Canada.

Nelson, W. R., Hirayama, H., and Rogers, D. W. O. (1985). The EGS4 Code System. Report SLAC-265, Stanford Linear Accelerator Center, Stanford, California.

Podgorsak, E. B. (Ed.) (2005). Radiation Oncology Physics: A Handbook for Teachers and Students. Vienna, Austria: International Atomic Energy Agency.

Rogers, D. W. O. (2006). Fifty years of Monte Carlo simulations for medical physics. Phys. Med. Biol., 51, $287-301$.

Rogers, D. W. O., and Bielajew, A. F. (1990). Monte Carlo techniques of electron and photon transport for radiation dosimetry. In K. R. Kase, B. E. Bjärngard, and F. H. Attix (Eds.) The Dosimetry of Ionizing Radiation, Vol III, (pp. 427 - 539). San Diego: Academic Press.

Rogers, D. W. O., Faddegon, B. A., Ding, G. X., Ma, C.-M., Wei, J., and Mackie, T. R. (1995). BEAM: A Monte Carlo code to simulate radiotherapy treatment units. Med. Phys., 22, $503-524$.

Rogers, D. W. O., Kawrakow, I., Seuntjens, J. P., Walters, B. R. B., and Mainegra-Hing, E. (2005a). NRC User Codes for EGSnrc. Technical Report PIRS-702(Rev B), National Research Council of Canada, Ottawa, Canada. 
Rogers, D. W. O., Walters, B., and Kawrakow, I. (2005b). BEAMnrc Users Manual. Technical Report PIRS-509(a)(Rev I), National Research Council of Canada, Ottawa, Canada.

Rossi, B., and Greisen, K. (1941). Cosmic-Ray Theory . Reviews of Modern Physics, 13, $241-309$.

RSICC Computer Code Collection (2002). Monte Carlo N-particle transport code system for multiparticle and high energy applications. Technical Report CCC-715, Los Alamos National Laboratory, Los Alamos.

Salvat, F., Fernández-Varea, J., and Sempau, J. (2003). PENELOPE, A Code System for Monte Carlo Simulation of Electron and Photon Transport. Tech. rep., Nuclear Energy Agency - Organisation for Economic Co-operation and Development, Paris.

Salvat, F., Jablonski, A., and Powell, C. (2005). ELSEPA-Dirac partial-wave calculation of elastic scattering of electrons and positrons by atoms, positive ions and molecules. Computer Physics Communications, 165(2), 157-190.

Sheikh-Bagheri, D., and Rogers, D. W. O. (2002). Sensitivity of megavoltage photon beam Monte Carlo simulations to electron beam and other parameters. Med. Phys., 29, $379-390$.

Sheikh-Bagheri, D., Rogers, D. W. O., Ross, C. K., and Seuntjens, J. P. (2000). Comparison of measured and Monte Carlo calculated dose distributions from the NRC linac. Med. Phys., 27, $2256-2266$.

Tonkopi, E., McEwen, M., Walters, B., and Kawrakow, I. (2005). Influence of ion chamber response on in-air profile measurements in megavoltage photon beams. Med. Phys., $32,2918-2927$. 
Vilches, M., García-Pareja, S., Guerrero, R., Anguiano, M., and Lallena, A. M. (2007). Monte Carlo simulation of the electron transport through thin slabs: A comparative study of PENELOPE, GEANT3, GEANT4, EGSnrc and MCNPX. Nucl. Inst. Meth. in Phys. Res. B, 254(2), 219-230. 